\title{
STRUCTURE OF CROSSED PRODUCTS BY STRICTLY PROPER ACTIONS ON CONTINUOUS-TRACE ALGEBRAS
}

\author{
SIEGFRIED ECHTERHOFF AND DANA P. WILLIAMS
}

\begin{abstract}
We examine the ideal structure of crossed products $B \rtimes_{\beta} G$ where $B$ is a continuous-trace $C^{*}$-algebra and the induced action of $G$ on the spectrum of $B$ is proper. In particular, we are able to obtain a concrete description of the topology on the spectrum of the crossed product in the cases where either $G$ is discrete or $G$ is a Lie group acting smoothly on the spectrum of $B$.
\end{abstract}

\section{INTRODUCTION}

A first step in understanding the structure of a crossed product $B \rtimes_{\beta} G$ is to describe its spectrum together with its Jacobson topology. Unfortunately, such a task is hopelessly out of reach in any sort of general setting. If we at least assume that the action of $G$ on the spectrum of $B$ is well behaved, then the Mackey-RieffelGreen machine allows us to describe $\left(B \rtimes_{\beta} G\right)^{\wedge}$ as a set in terms of projective representations of the stability groups for the action of $G$ on the spectrum of $B$. These representations are determined by the Mackey obstructions which can vary wildly even in the seemingly most benign situations. This wild behavior is often an insurmountable obstruction to describing the Jacobson topology. In the case where one assumes away the difficulty - that is, where we assume all the Mackey obstructions vanish - there has been considerable progress if it is also assumed that the stabilizers are constant (see, for example [19, 21, 23, 26]) and when the stabilizer map is continuous 25]. When nontrivial Mackey obstructions are allowed, progress has been more modest and is usually accompanied with robust hypotheses [5, 6, 9, 10, 14. In this article, we take on the case where $G$ acts properly on the spectrum $X$ of a continuous-trace $C^{*}$-algebra $B$. This in particular guarantees that the Mackey-Rieffel-Green machine works very nicely and gives us a tidy settheoretic description of $\left(B \rtimes_{\beta} G\right)^{\wedge}$. Specifically, if $\left[\omega_{x}\right] \in H^{2}\left(G_{x}, \mathbb{T}\right)$ is the Mackey obstruction at $x \in X$, then we can form the set

$$
\operatorname{Stab}\left(X_{\beta}\right)^{\wedge}=\left\{(x, \sigma): x \in X \text { and } \sigma \in \widehat{G}_{x, \omega_{x}}\right\},
$$

where $\widehat{G}_{x, \omega_{x}}$ is the collection of irreducible $\omega_{x}$-representations of the stability group $G_{x}$. Then $\operatorname{Stab}\left(X_{\beta}\right)^{\wedge}$ carries a natural $G$-action and we can identify the orbit space $G \backslash \operatorname{Stab}\left(X_{\beta}\right)^{\wedge}$ with the spectrum of the crossed product via an induction process: $(x, \sigma) \mapsto \operatorname{ind}_{x}(\sigma)$. Our first result is to equip $\operatorname{Stab}\left(X_{\beta}\right)^{\wedge}$ with a natural topology so that induction induces a homeomorphism with the spectrum.

Received by the editors August 21, 2012.

2010 Mathematics Subject Classification. Primary 46L55.

The research for this paper was partially supported by the German Research Foundation (SFB 478 and SFB 878) and the EU-Network Quantum Spaces Noncommutative Geometry (Contract No. HPRN-CT-2002-00280) as well as the Edward Shapiro Fund at Dartmouth College. 
However to give a more useful and concrete description of the topology on $\operatorname{Stab}\left(X_{\beta}\right)^{\wedge}$, and hence on the spectrum of the crossed product, we require some hypotheses on $G$ (to at least partially tame the Mackey obstructions). In our main results, we are able to give such descriptions in the cases where (1) $G$ is discrete, and (2) when $X$ is a manifold and $G$ is a Lie group acting smoothly, as well as properly, on $X$. For example, in the case where $G$ is discrete, we can show that $\left(x_{n}, \sigma_{n}\right) \rightarrow(x, \sigma)$ in $\operatorname{Stab}\left(X_{\beta}\right)^{\wedge}$ if and only if $x_{n} \rightarrow x$ in $X$ and we eventually have $G_{x_{n}} \subseteq G_{x}$ and $\sigma_{n}$ equivalent to a subrepresentation of $\left.\sigma\right|_{G_{x_{n}}}$. (The result for Lie groups is similar, but a bit more technical.)

Since the methods of proof in our main results seem to require separability, we assume throughout that the spaces and groups that appear are second countable and that our algebras are separable. Representations and other homomorphisms between $C^{*}$-algebras are assumed to be $*$-preserving.

The starting point for this article is a nice structure theorem, in terms of a generalized fixed point algebra, for crossed products for proper actions on spaces due to the first author and H. Emerson [8. In fact, we make considerable use of some of the results in that paper as well as some ideas from the Ph.D. thesis of Katharina Neumann [18. In $\S 1$, we review the construction of the generalized fixed point algebra from [8] as well as some preliminary results on $C^{*}$-bundles. In $\S 2$, we specialize to the case of strictly proper actions on continuous-trace $C^{*}$-algebras and study in detail the fixed point algebras that arise in our investigations. We also state the particular version of the Mackey-Rieffel-Green machine that we will employ. In $\S 3$, we introduce the topology on $\operatorname{Stab}\left(X_{\beta}\right)^{\wedge}$, and show that the spectrum of $B \rtimes_{\beta} G$ is the quotient. Our main results are stated and proved in $\S 4$. In $\S 5$, we exhibit a class of group extensions - namely compact extensions $G$ of abelian groups where we can apply our results to the spectrum of $G$. In particular, we work out in detail the spectrum of the crystallographic group p4g. Even in this case, our description is very subtle and clearly demonstrates the difficulty of working with varying Mackey obstructions.

\section{The Bundle StRUCture of AN $X \rtimes G$-ALGEBRA}

Recall that we call a $C^{*}$-algebra $B$ a $C_{0}(X)$-algebra if there is a nondegenerate map $\Phi: C_{0}(X) \rightarrow Z M(B)$. For the basics on $C_{0}(X)$-algebras and their associated upper-semicontinuous $C^{*}$-bundles, we refer to [30, Appendix $\left.\mathrm{C}\right]$. For convenience, we recall some standard facts and notation here. In particular, every $C_{0}(X)$-algebra $B$ is $C_{0}(X)$-isomorphic to the section algebra $\Gamma_{0}(X, \mathscr{B})$ of an upper-semicontinuous $C^{*}$-bundle $p: \mathscr{B} \rightarrow X$. The fibre over $x$ can be identified with the quotient $B(x)=B / I_{x}$, where $I_{x}$ is the ideal of $B$ generated by products $f \cdot a$ with $f \in C_{0}(X)$ such that $f(x)=0$ (and where, as is standard, we have written $f \cdot a$ in place of $\Phi(f)(a)$ ). When the map $b \mapsto\|b\|$ is continuous from $\mathscr{B}$ to $\mathbb{R}$ (or equivalently, when the map $x \mapsto\|b(x)\|$ is continuous for $b \in B)$, we say that $\mathscr{B}$ is a continuous $C^{*}$-bundle. If $Y$ is a closed subset of $X$ and if $B=\Gamma_{0}(X, \mathscr{B})$ is a $C_{0}(X)$-algebra, then $B_{Y}:=\Gamma_{0}\left(Y,\left.\mathscr{B}\right|_{Y}\right)$ is the restriction of $B$ to $Y$. We can also realize $B_{Y}$ as the quotient of $B$ by the ideal generated by $C_{0}(X \backslash Y) \cdot B$.

As in [8], if $B$ is a $C_{0}(X)$-algebra for a $G$-space $X$, and if $\beta: G \rightarrow$ Aut $B$ is a $C_{0}(X)$-linear dynamical system, then we call $B$ an $X \rtimes G$-algebra if the structure map $\Phi: C_{0}(X) \rightarrow Z M(B)$ is $G$-equivariant. 
In this paper, we will always assume that the action of $G$ on $X$ is proper in the sense that the map $(s, x) \mapsto(s \cdot x, x)$ is a proper map from $G \times X$ to $X \times X$. If $X$ is a proper $G$-space, then the orbit space $G \backslash X$ is Hausdorff and the stability groups $G_{x}=\{s \in G: s \cdot x=x\}$ are compact [20, Theorem 1.2.9 and Proposition 1.1.4].

If $B$ is an $X \rtimes G$-algebra for a proper $G$-action - in which case we say that $B$ is a proper $X \rtimes G$-algebra - then there is an associated generalized fixed point algebra $B^{G, \beta}$ which agrees with the usual fixed point algebra when $G$ is compact $([8, \S 2])$. We sketch the details of its construction here. We first form the induced algebra $C_{0}\left(X \times_{G, \beta} B\right)$ which consists of continuous functions $F: X \rightarrow B$ which have the property $F(s \cdot x)=\beta_{s}(F(x))$ for all $s \in G$ and $x \in X$ and such that $G \cdot x \mapsto\|F(x)\|$ vanishes at infinity on $G \backslash X$. (Induced algebras have been studied extensively for free and proper actions of $G$ on $X$ - for example see [24, 22] and [13] - but other than [8, Lemma 2.2], we are not aware of any systematic study of induced algebras for proper actions that are not necessarily free.) The properness of the $G$-action on $X$ guarantees that the diagonal action of $G$ on $X \times X$ is proper. Hence the orbit space $G \backslash(X \times X)$ is Hausdorff and we obtain a nondegenerate homomorphism

$$
\Phi: C_{0}(G \backslash(X \times X)) \rightarrow Z M\left(C_{0}\left(X \times_{G, \beta} B\right)\right)
$$

by $\Phi(f)(F)(x)(y)=f([x, y]) F(x)(y)$. Then the generalized fixed point algebra $B^{G, \beta}$ is defined to be the restriction of the $C_{0}(G \backslash(X \times X))$-algebra $C_{0}\left(X \times_{G, \beta} B\right)$ to the closed subspace $G \backslash \Delta(X)$. After identifying $X$ with $\Delta(X)$, we see that $B^{G, \beta}$ inherits a $C_{0}(G \backslash X)$-algebra structure. As mentioned above, if $G$ is compact, then the generalized fixed point algebra $B^{G, \beta}$ is just the usual fixed point algebra. Also, if $B=C_{0}(X, A)$ for some $C^{*}$-algebra $A$ admitting a $G$-action $\beta$, then $C_{0}(X, A)^{G, \mathrm{lt} \otimes \beta}$ is just the induced algebra $C_{0}\left(X \times_{G, \beta} A\right)$.

If $G_{x}$ is the stabilizer of $x$, then $I_{x}=C_{0}(X \backslash\{x\}) \cdot B$ is a $G_{x}$-invariant ideal of $B$ and we have an induced action $\beta^{x}: G_{x} \rightarrow$ Aut $B(x)$ given by $\beta_{s}^{x}(b(x))=\beta_{s}(b)(x)$. In fact, if $B=\Gamma_{0}(X, \mathscr{B})$, then $G$ acts on $\mathscr{B}$ via $\beta_{x, s}: B(x) \rightarrow B(s \cdot x)$ given by

$$
\beta_{x, s}(b(x))=\beta_{s}(b)(s \cdot x) \quad \text { for all } b \in B .
$$

Then

$$
\beta_{s \cdot x, h} \circ \beta_{s, x}=\beta_{x, s h} \quad \text { for all } s, h \in G \text { and } x \in X .
$$

If $h \in G_{x}$, then $\beta_{x, h}$ is just the induced automorphism $\beta_{h}^{x}$ as defined above 1

Lemma 1.1. Let $B$ be a proper $X \rtimes G$-algebra. Then the fibre over $G \cdot x$ of the $C_{0}(G \backslash X)$-algebra $B^{G, \beta}$ is isomorphic to $B(x)^{G_{x}, \beta^{x}}$. In fact, we can realize $B^{G, \beta}$ as

$$
\begin{aligned}
\left\{b \in \Gamma_{b}(X, \mathscr{B}): b(s \cdot x)=\right. & \beta_{s}^{x}(b(x)) \text { for all } s \in G \text { and } x \in X, \\
& \text { and such that } \left.G \cdot x \mapsto\|b(x)\| \text { is in } C_{0}(G \backslash X)\right\} .
\end{aligned}
$$

Then the isomorphism of the fibre of $B^{G, \beta}$ over $G \cdot x$ with $B(x)^{G_{x}, \beta^{x}}$ is given via evaluation at $x$. If $\mathscr{B}$ is a continuous $C^{*}$-bundle, then $B^{G, \beta}$ is the section algebra of a continuous $C^{*}$-bundle over $G \backslash X$.

\footnotetext{
${ }^{1}$ The $\beta_{x, s}$ give an action of the transformation groupoid $X \rtimes G$ on $\mathscr{B}$ which explains our terminology and notation for $X \rtimes G$-algebras. We will not make use of groupoid technology in this paper.
} 
Proof. By [8, Lemma 2.2], the fibre $C_{0}\left(X \times_{G, \beta} B\right)(G \cdot x)$ is isomorphic to the fixed point algebra $B^{G_{x}, \beta}$ via evaluation at $x$. On the other hand, the $(G \cdot x)$-fibre of $B^{G, \beta}$ is $\left\{F(x)(x): F \in C_{0}\left(X \times_{G, \beta} B\right)\right\}$. Since $B^{G_{x}, \beta}=\left\{F(x): F \in C_{0}\left(X \times_{G, \beta} B\right)\right\}$, we just need to see that evaluation at $x$ takes $B^{G_{x}, \beta}$ onto $B(x)^{G_{x}, \beta^{x}}$. (It clearly takes values in $B(x)^{G_{x}, \beta^{x}}$.) However, if $b_{x} \in B(x)^{G_{x}, \beta^{x}}$, then there is a $b \in B$ such that $b(x)=b_{x}$. But if $s \in G_{x}$, then $\beta_{s}(b)(x)=\left(\beta^{x}\right)_{s}(b(x))=b_{x}$. Thus we can let $\tilde{b}=\int_{G_{x}} \beta_{s}(b) d s$. Then $\tilde{b} \in B^{G_{x}, \beta}$ and $\tilde{b}(x)=b_{x}$.

Clearly, we can identify $C_{0}\left(X \times_{G, \beta} B\right)$ with continuous sections $F$ of the $C^{*}$ bundle $X \times \mathscr{B}$ over $X \times X$ such that

$$
\begin{aligned}
& F(s \cdot x, s \cdot y)=\beta_{s}(F(x, \cdot))(s \cdot y)=\beta_{y, s}(F(x, y)) \quad \text { and such that } \\
& G \cdot x \mapsto \sup _{y}\|F(x, y)\| \quad \text { vanishes at infinity on } G \backslash X .
\end{aligned}
$$

On the other hand, (1.3) is a closed $C_{0}(G \backslash X)$-subalgebra $D$ of $\Gamma_{b}(X, \mathscr{B})$. The map sending $F$ to $(x \mapsto F(x, x))$ defines a homomorphism of $C_{0}\left(X \times_{G, \beta} B\right)$ into $D$ which factors through a $C_{0}(G \backslash X)$-homomorphism $\psi$ of $B^{G, \beta}$ into $D$. Then $\psi$ induces a map of the fibre $B^{G, \beta}(G \cdot x)$ into $D(G \cdot x)$. Evaluation at $x$ clearly identifies $D(G \cdot x)$ with a subalgebra of $B(x)^{G_{x}, \beta^{x}}$, and the first part of the lemma implies that the image is all of $B(x)^{G_{x}, \beta^{x}}$ and that $\psi$ induces an isomorphism of the fibres over $G \cdot x$. Hence $\psi$ is an isomorphism so that we can identify $D$ with $B^{G, \beta}$, as claimed.

To verify the statement about continuous bundles, it suffices to see that $G \cdot x \mapsto$ $\|b(x)\|$ is continuous for $b$ in (1.3). But this is automatic if $\mathscr{B}$ is a continuous bundle.

If $B$ is a proper $X \rtimes G$-algebra, then so is $B \otimes \mathcal{K}\left(L^{2}(G)\right)$ with $C_{0}(X)$ acting on the first factor and with respect to the diagonal $G$-action $\beta \otimes \operatorname{Ad} \rho$, where $\rho: G \rightarrow$ $U\left(L^{2}(G)\right)$ is the right regular representation. (When $\mathcal{H}$ is a Hilbert space, we will write $U(\mathcal{H})$ for the unitaries on $\mathcal{H}$ equipped with the strong operator topology.) Clearly, $B \otimes \mathcal{K}\left(L^{2}(G)\right)$ is the section algebra of a bundle $\mathscr{B} \otimes \mathcal{K}\left(L^{2}(G)\right)$ over $X$ built from the fibres $B(x) \otimes \mathcal{K}\left(L^{2}(G)\right)$ and for which $x \mapsto b(x) \otimes T$ is a continuous section for every $b \in B$ and $T \in \mathcal{K}\left(L^{2}(G)\right)$. Since we can identify $\operatorname{Prim} B$ and $\operatorname{Prim}\left(B \otimes \mathcal{K}\left(L^{2}(G)\right)\right)$, it follows from [15] or [30, Theorem C.26] that $\mathscr{B} \otimes \mathcal{K}\left(L^{2}(G)\right)$ is continuous when $\mathscr{B}$ is continuous. Hence we can quote [8, Theorem 2.14] to deduce the following.

Theorem 1.2 ([8, Theorem 2.14]). Let $B$ be a proper $X \rtimes G$-algebra. Then $B \rtimes_{\beta} G$ is isomorphic to the generalized fixed point algebra $\left(B \otimes \mathcal{K}\left(L^{2}(G)\right)\right)^{G, \beta \otimes \operatorname{Ad} \rho}$, which is a $C_{0}(G \backslash X)$-algebra with fibres over $G \cdot x$ isomorphic to

$$
\left(B(x) \otimes \mathcal{K}\left(L^{2}(G)\right)\right)^{G_{x}, \beta^{x} \otimes \operatorname{Ad} \rho} .
$$

Moreover if $B$ is (the section algebra of a) continuous bundle of $C^{*}$-algebras, then so is $\left(B \otimes \mathcal{K}\left(L^{2}(G)\right)\right)^{G, \beta \otimes \operatorname{Ad} \rho}$.

\section{Proper actions on $C^{*}$-Algebras with COntinuous trace}

Now we restrict our attention to the situation where $B$ is a continuous-trace $C^{*}$-algebra with spectrum $X$. Then we may assume that $B$ is the section algebra $\Gamma_{0}(X, \mathscr{B})$ of a continuous $C^{*}$-bundle $\mathscr{B}$ with fibres $B(x)=\mathcal{K}\left(\mathcal{H}_{x}\right)$ for Hilbert spaces 
$\mathcal{H}_{x}$. We will also assume that we have a dynamical system $\beta: G \rightarrow$ Aut $B$ such that the induced action of $G$ on $X$ is proper. In the sequel, we will simply say that $G$ acts strictly properly on $B$. We use the term "strictly properly" to make sure that our notion is not mistaken with the much weaker notion of proper actions on $C^{*}$-algebras due to Rieffel [27, 28].

Recall that $\operatorname{Aut} \mathcal{K}(\mathcal{H})$ can be identified with the projective unitary group $U(\mathcal{H}) /$ $\mathbb{T} 1$ in a canonical way, and we can view $\beta^{x}: G_{x} \rightarrow$ Aut $B(x)$ as a continuous homomorphism of $G_{x}$ into $P U\left(\mathcal{H}_{x}\right)$. (Thus, $\beta^{x}$ is often called a projective representation of $G_{x}$ on $\mathcal{H}_{x} \cdot \sqrt{2}$ Since each $\mathcal{H}_{x}$ is separable, there is a Borel map $V_{x}: G_{x} \rightarrow U\left(\mathcal{H}_{x}\right)$ such that $\beta^{x}=\operatorname{Ad} V_{x}$. Our choice of $V_{x}$ determines a Borel 2-cocycle $\omega_{x}: G \times G \rightarrow \mathbb{T}$ such that

$$
V_{x}(s) V_{x}(t)=\omega_{x}(s, t) V_{x}(s t) \text { for all } s, t \in G_{x} .
$$

Assuming, as we do, that we have arranged that $V_{x}(e)=1_{\mathcal{H}_{x}}$, then our cocycle is normalized in that $\omega_{x}(e, s)=1=\omega_{x}(s, e)$ for all $s \in G$. A normalized 2-cocycle is called a multiplier on $G$. The class $\left[\omega_{x}\right] \in H^{2}\left(G_{x}, T\right)$ depends only on $\beta_{x}$ and represents the obstruction to $\beta_{x}$ being implemented by a unitary representation of $G_{x}$ on $\mathcal{H}_{x}$. It is called the Mackey obstruction at $x$.

Our task in this section is to examine the structure of the fibres $(B(x) \otimes$ $\left.\mathcal{K}\left(L^{2}(G)\right)\right)^{G_{x}, \beta^{x} \otimes \operatorname{Ad} \rho}$ of $\left(B \otimes \mathcal{K}\left(L^{2}(G)\right)\right)^{G, \beta \otimes \operatorname{Ad} \rho}$. Then if $B(x)=\mathcal{K}\left(\mathcal{H}_{x}\right)$ and $\beta^{x}=\operatorname{Ad} V_{x}$ as above, we want to examine $\mathcal{K}\left(\mathcal{H}_{x} \otimes L^{2}(G)\right)^{G_{x}, \operatorname{Ad}\left(V_{x} \otimes \rho\right)}$. Thus, dropping the " $x$ ", we want to consider the fixed point algebra

$$
\mathcal{K}\left(\mathcal{H} \otimes L^{2}(G)\right)^{K, \operatorname{Ad}(V \otimes \rho)},
$$

where $K$ is a compact subgroup of $G$ and where $V: K \rightarrow U(\mathcal{H})$ is a Borel lift of a projective representation $\beta: K \rightarrow P U(\mathcal{H})$. We let $\omega \in H^{2}(K, \mathbb{T})$ be the multiplier associated to $V$ as in (2.1).

A Borel map $\sigma: K \rightarrow U\left(\mathcal{H}_{\sigma}\right)$ which satisfies $\sigma(s) \sigma(t)=\omega(s, t) \sigma(s t)$ is called $a$ multiplier representation with multiplier $\omega$ or, more simply, an $\omega$-representation of $K$. We have two canonical $\omega$-representations of $K$ on $L^{2}(K)$, called the left- and right-regular $\omega$-representations, which are given, respectively, by

$$
\lambda_{K}^{\omega}(s) \xi(t)=\omega\left(s, s^{-1} t\right) \xi\left(s^{-1} t\right) \quad \text { and } \quad \rho_{K}^{\omega}(s) \xi(t)=\omega(t, s) \xi(t s) .
$$

The multiplier $\omega$ determines a group structure on $K \times \mathbb{T}$ :

$$
(s, z)(t, w)=(s t, \omega(s, t) z w) \text { and }(s, z)^{-1}=\left(s^{-1}, \overline{z \omega\left(s, s^{-1}\right)}\right) .
$$

A nontrivial result due to Mackey implies that the resulting group, denoted by $K \times_{\omega} \mathbb{T}$, has a compact topology (since $K$ is compact) such that $K \times_{\omega} \mathbb{T}$ is a central extension of $K$ by $\mathbb{T}$. (For references and more details, see page 376 of [30].) Furthermore, the $\omega$-representations of $K$ are then in one-to-one correspondence with the unitary representations of $K \times_{\omega} \mathbb{T}$ whose restriction to $\mathbb{T}$ is a multiple of the identity character $z \mapsto z$ (see, for example, [30, Proposition D.28]). In a similar way, we get a correspondence between the $\bar{\omega}$-representations of $K$ and the unitary representations of $K \times_{\omega} \mathbb{T}$ that restrict to a multiple of the character $z \mapsto \bar{z}$ on $\mathbb{T}$. Since $K \times_{\omega} \mathbb{T}$ is compact, every irreducible $\omega$-representation of $K$ is finite-dimensional. We will write $\widehat{K}_{\omega}$ for the set of irreducible $\omega$-representations.

\footnotetext{
${ }^{2}$ For an elementary discussion of projective representations, see [30, §D.2].
} 
Remark 2.1. Using the above correspondence and the well-known theory of unitary representations of compact groups (for example, see [2, $\S \S 7.2-3]$ ), we see that the space $\mathcal{H}_{W}$ of any $\omega$-representation $W: K \rightarrow U\left(\mathcal{H}_{W}\right)$ on a separable Hilbert space decomposes as

$$
\mathcal{H}_{W}=\bigoplus_{\sigma \in \widehat{K}_{\omega}} \mathcal{H}(\sigma)
$$

where $\mathcal{H}(\sigma)$ is the isotope of $\sigma$. That is, $\mathcal{H}(\sigma)$ is the union of closed $K$-invariant subspaces $\mathcal{H}^{\prime}$ of $\mathcal{H}_{W}$ such that the restriction of $W$ to $\mathcal{H}^{\prime}$ is equivalent to $\sigma$. Then each $\mathcal{H}(\sigma)$ decomposes as a tensor product $\mathcal{H}_{\sigma}^{\prime} \otimes \mathcal{H}_{\sigma}$ in such a way that

$$
\mathcal{H}_{W} \cong \bigoplus_{\sigma \in \widehat{K}_{\omega}} \mathcal{H}_{\sigma}^{\prime} \otimes \mathcal{H}_{\sigma} \text { and } W \cong \bigoplus_{\sigma \in \widehat{K}_{\sigma}} 1_{\mathcal{H}_{\sigma}^{\prime}} \otimes \sigma
$$

Then, and this is the point, the fixed point algebra is given by

$$
\mathcal{K}\left(\mathcal{H}_{W}\right)^{K, \operatorname{Ad} W} \cong \bigoplus_{\sigma \in \widehat{K}_{\omega}} \mathcal{K}\left(\mathcal{H}_{\sigma}^{\prime}\right) \otimes 1_{\mathcal{H}_{\sigma}} .
$$

Therefore to understand the structure of $\mathcal{K}\left(\mathcal{H} \otimes L^{2}(G)\right)^{K, \operatorname{Ad} V \otimes \rho}$, we need to decompose the Hilbert space $\mathcal{H} \otimes L^{2}(G)$ into isotopes as above via an analogue of the Peter-Weyl Theorem for compact groups. Although the result is certainly well known, we include a statement and proof as we lack a direct reference. First, for any $\omega$-representation $\sigma: K \rightarrow U\left(\mathcal{H}_{\sigma}\right)$, we write $\mathcal{H}_{\sigma}^{*}$ for the conjugate Hilbert space and let $\sigma^{*}: K \rightarrow U\left(\mathcal{H}_{\sigma}^{*}\right)$ be the conjugate representation of $\sigma: \sigma^{*}(s)\left(v^{*}\right)=(\sigma(s)(v))^{*}$. Then $\sigma^{*}$ is a $\bar{\omega}$-representation and the map $\sigma \mapsto \sigma^{*}$ is a bijection between $\widehat{K}_{\omega}$ and $\widehat{K}_{\bar{\omega}}$. There is a unique linear map

$$
\mathcal{H}_{\sigma} \otimes \mathcal{H}_{\sigma}^{*} \rightarrow L^{2}(K)
$$

sending the elementary tensor $v \otimes w^{*}$ to $g_{v, w}^{\sigma}$, where $g_{v, w}^{\sigma}(k)=\sqrt{d_{\sigma}}(v \mid \sigma(k) w)$ and $d_{\sigma}=\operatorname{dim} \mathcal{H}_{\sigma}$.

Lemma 2.2 (Peter-Weyl). Let $\omega$ be a multiplier on the compact group $K$. Then the maps (2.2) above induce a Hilbert-space isomorphism of

$$
\bigoplus_{\sigma \in \widehat{K}_{\omega}} \mathcal{H}_{\sigma} \otimes \mathcal{H}_{\sigma}^{*} \quad \text { with } \quad L^{2}(K)
$$

which intertwines the representation $\bigoplus_{\sigma} \sigma \otimes 1_{\mathcal{H}_{\sigma}^{*}}$ with the left regular $\omega$-representation $\lambda_{K}^{\omega}$, as well as the representation $\bigoplus_{\sigma} 1_{\mathcal{H}_{\sigma}} \otimes \sigma^{*}$ with the right regular $\bar{\omega}$-representation $\rho_{K}^{\bar{\omega}}$.

Proof. As we will see, the result follows readily from the classical Peter-Weyl Theorem for the compact group $K \times_{\omega} \mathbb{T}$ which gives us the decomposition

$$
L^{2}\left(K \times_{\omega} \mathbb{T}\right) \cong \bigoplus_{\tau \in\left(K \times_{\omega} \mathbb{T}\right)^{\wedge}} \mathcal{H}_{\tau} \otimes \mathcal{H}_{\tau}^{*}
$$

in which an elementary tensor $v \otimes w^{*}$ in $\mathcal{H}_{\tau} \otimes \mathcal{H}_{\tau}^{*}$ corresponds to the function $f_{v, w}^{\tau}$ given by

$$
f_{v, w}^{\tau}(k, z):=\sqrt{d_{\tau}}(v \mid \tau(k, z) w)
$$


Since $\mathbb{T}$ is central in $K \times_{\omega} \mathbb{T}$, the restriction to $\mathbb{T}$ of any irreducible representation $\tau$ of $K \times_{\omega} \mathbb{T}$ must be a multiple of a character $\chi_{n} \in \widehat{\mathbb{T}}$ of the form $\chi_{n}(z)=z^{n}$ (with $n \in \mathbb{Z})$. Thus $\tau(k, z)=\tau((e, z)(k, 1))=\chi_{n}(z) \tau(k, 1)$, and

$$
f_{v, w}^{\tau}(k, z)=\bar{\chi}_{n}(z) g_{v, w}^{\tau}(k) \quad \text { where } \quad g_{v, w}^{\tau}(k):=\sqrt{d_{\tau}}(v \mid \tau(k, 1) w) .
$$

Recall that if $\left\{v_{1}^{\tau}, \ldots, v_{n_{\tau}}^{\tau}\right\}$ is a basis for $\mathcal{H}_{\tau}$ and if we let $f_{i j}^{\tau}=f_{v_{i}, v_{j}}^{\tau}$, then

$$
\left\{f_{i j}^{\tau}: \tau \in\left(K \times_{\omega} \mathbb{T}\right)^{\wedge} \text { and } 1 \leq i, j \leq n_{\tau}\right\}
$$

is an orthonormal basis for $L^{2}\left(K \times_{\omega} \mathbb{T}\right)$. Thus if we let $g_{i j}^{\tau}(k)=f_{i j}^{\tau}(k, 1)$ and $\left(K \times_{\omega} \mathbb{T}\right)_{n}^{\wedge}$ be the collection of those $\tau$ which restrict to a multiple of $\chi_{n}$ on $\mathbb{T}$, then we get an orthonormal basis of $L^{2}(K) \otimes L^{2}(\mathbb{T}) \cong L^{2}\left(K \times_{\omega} \mathbb{T}\right)$ of the form

$$
\bigcup_{n \in \mathbb{Z}}\left\{g_{i j}^{\tau} \otimes \bar{\chi}_{n}: \tau \in\left(K \times_{\omega} \tau\right)_{n}^{\wedge} \text { and } 1 \leq i, j \leq n_{\tau}\right\} .
$$

Since $\left\{\bar{\chi}_{n}: n \in \mathbb{Z}\right\}$ is an orthonormal basis for $L^{2}(\mathbb{T})$, we can conclude that for each $n \in \mathbb{Z}$,

$$
\left\{g_{i j}^{\tau}: \tau \in\left(K \times_{\omega} \mathbb{T}\right)_{n}^{\wedge} \text { and } 1 \leq i, j \leq n_{\tau}\right\}
$$

is an orthonormal basis for $L^{2}(K)$. In the case $n=1$ we get a decomposition as in the lemma.

We still need to see that this isomorphism intertwines the given representations. But the computation

$$
\begin{aligned}
\left(\lambda_{K}^{\omega}(l) g_{v, w}^{\sigma}\right)(k) & =\omega\left(l, l^{-1} k\right) g_{v, w}^{\sigma}\left(l^{-1} k\right) \\
& =\omega\left(l, l^{-1} k\right) \sqrt{d_{\sigma}}\left(v \mid \sigma\left(l^{-1} k\right) w\right)
\end{aligned}
$$

which, since $\sigma$ is an $\omega$-representation, is

$$
=\omega\left(l, l^{-1} k\right) \omega\left(l^{-1}, k\right) \sqrt{d_{\sigma}}\left(v \mid \sigma\left(l^{-1}\right) \sigma(k) w\right)
$$

which, since $\sigma\left(l^{-1}\right)=\omega\left(l, l^{-1}\right) \sigma(l)^{*}$, is

$$
=\omega\left(l, l^{-1} k\right) \omega\left(l^{-1}, k\right) \bar{\omega}\left(l, l^{-1}\right) \sqrt{d_{\sigma}}\left(v \mid \sigma(l)^{*} \sigma(k) w\right)
$$

which, since the cocycle identity implies $\omega\left(l, l^{-1} k\right) \omega\left(l^{-1}, k\right) \bar{\omega}\left(l, l^{-1}\right)=\omega(e, k)=1$, is

$$
\begin{aligned}
& =\sqrt{d_{\sigma}}(\sigma(l) v \mid \sigma(k) w) \\
& =g_{\sigma(l) v, w}^{\sigma}(k),
\end{aligned}
$$

shows that this isomorphism intertwines $\bigoplus_{\sigma} \sigma \otimes 1_{\mathcal{H}_{\sigma}^{*}}$ and the left-regular $\omega$-representation $\lambda_{K}^{\omega}$. The computation involving the right-regular $\omega$ representation is similar, but less messy.

Remark 2.3. We can apply the above lemma to $\bar{\omega}$ and then use the bijection between $\widehat{K}_{\omega}$ and $\widehat{K}_{\bar{\omega}}$ to obtain a decomposition $L^{2}(K)$ with $\bigoplus_{\sigma \in \widehat{K}_{\omega}} \mathcal{H}_{\sigma}^{*} \otimes \mathcal{H}_{\sigma}$ which intertwines $\bigoplus_{\sigma} \sigma^{*} \otimes 1_{\mathcal{H}_{\sigma}}$ with $\lambda_{K}^{\bar{\omega}}$ and $\bigoplus_{\sigma} 1_{\mathcal{H}_{\sigma}^{*}} \otimes \sigma$ with $\rho_{K}^{\omega}$. This is the decomposition we will employ below.

We will need a twisted version of the absorption principle for regular representations of $G$. The proof is a straightforward calculation which we omit. 
Lemma 2.4. Suppose that $\omega$ and $\mu$ are multipliers on a locally compact group $G$. Let $V: G \rightarrow U(\mathcal{H})$ be an $\omega$-representation of $G$ and $W: L^{2}(G, \mathcal{H}) \rightarrow L^{2}(G, \mathcal{H})$ the unitary operator given by $(W \xi)(s)=V_{s} \xi(s)$ for $\xi \in L^{2}(G, \mathcal{H})$ and $s \in G$. Then for all $s \in G$,

$$
W\left(V \otimes \rho_{G}^{\mu}\right)(s) W^{*}=1_{\mathcal{H}} \otimes \rho_{G}^{\omega \mu}(s) \quad \text { and } \quad W^{*}\left(V \otimes \lambda_{G}^{\mu}\right)(s) W=1_{\mathcal{H}} \otimes \lambda_{G}^{\omega \mu}(s) .
$$

In particular, $V \otimes \rho_{G} \cong 1_{\mathcal{H}} \otimes \rho_{G}^{\omega}$ and $V \otimes \lambda_{G} \cong 1_{H} \otimes \lambda_{G}^{\omega}$.

Now let our compact group $K$ be a subgroup of a locally compact group $G$. If $\tau$ is a unitary representation of $K$ on $\mathcal{H}_{\tau}$, then the induced representation $U^{\tau}$ acts by left-translation on the Hilbert space $L^{2}\left(G \times_{K, \tau} \mathcal{H}\right)$ of (almost everywhere equivalence classes of) square integrable functions from $G$ to $\mathcal{H}_{\tau}$ satisfying $\xi(s k)=$ $\tau\left(k^{-1}\right)(\xi(s))$ for all $s \in G$ and $k \in K$. Alternatively, we can realize $L^{2}\left(G \times{ }_{K, \tau}\right.$ $\mathcal{H})$ as $L^{2}$-sections of the vector bundle $K \backslash\left(G \times \mathcal{H}_{\tau}\right)$ for the diagonal action $k$. $(g, v)=\left(g k^{-1}, \tau(k)(v)\right)$. In particular, the isomorphism $U: L^{2}(G) \rightarrow L^{2}\left(G \times_{K, \lambda_{K}}\right.$ $\left.L^{2}(K)\right)$, given by $(U \xi)(s)(k)=\xi(s k)$ for $s \in G$ and $k \in K$, intertwines the leftregular representation $\lambda_{G}$ of $G$ with $U^{\lambda_{K}}$. It also intertwines the restriction of the right-regular representation $\left.\rho_{G}\right|_{K}$ with the pointwise action of the right-regular representation $\rho_{K}$ of $K$ on the elements of $L^{2}\left(G \times_{K, \lambda_{K}} L^{2}(K)\right)$.

Thus if $V: K \rightarrow U(\mathcal{H})$ is an $\omega$-representation, we get a unitary

$$
\tilde{U}: \mathcal{H} \otimes L^{2}(G) \rightarrow L^{2}\left(G \times_{K, 1_{\mathcal{H}} \otimes \lambda_{K}}\left(\mathcal{H} \otimes L^{2}(K)\right)\right)
$$

which intertwines the representation $1_{\mathcal{H}} \otimes \lambda_{G}$ of $G$ on $\mathcal{H} \otimes L^{2}(G)$ with the induced representation $U^{1_{\mathcal{H}} \otimes \lambda_{K}}$, as well as the representation $\left.V \otimes \rho_{G}\right|_{K}$ with the representation of $K$ given by the pointwise action of $V \otimes \rho_{K}$ on elements of $L^{2}\left(G \times_{K, 1_{\mathcal{H}} \otimes \lambda_{K}}\right.$ $\left.\left(\mathcal{H} \otimes L^{2}(K)\right)\right)$.

Let $W$ be the unitary on $L^{2}(K, \mathcal{H})$ from Lemma 2.4 which intertwines $1_{\mathcal{H}} \otimes \lambda_{K}$ with $V \otimes \lambda_{K}^{\bar{\omega}}$ and $V \otimes \rho_{K}$ with $1_{\mathcal{H}} \otimes \rho_{K}^{\omega}$. Then, after identifying $\mathcal{H} \otimes L^{2}(K)$ with $L^{2}(K, \mathcal{H})$, we can apply $W$ pointwise to elements in $L^{2}\left(G \times_{K, 1_{\mathcal{H}} \otimes \lambda_{K}}\left(\mathcal{H} \otimes L^{2}(K)\right)\right)$ to obtain a unitary

$$
\tilde{W}: L^{2}\left(G \times_{K, 1_{\mathcal{H}} \otimes \lambda_{K}}\left(\mathcal{H} \otimes L^{2}(K)\right)\right) \rightarrow L^{2}\left(G \times_{K, V \otimes \lambda_{K}^{\bar{\omega}}}\left(\mathcal{H} \otimes L^{2}(K)\right)\right)
$$

which intertwines the induced representations $U^{1 \mathcal{H} \otimes \lambda_{K}}$ and $U^{V \otimes \lambda_{K}^{\bar{\omega}}}$ of $G$, and the pointwise acting $\omega$-representation $V \otimes \rho_{K}$ with the pointwise acting $\omega$-representation $1 \otimes \rho_{K}^{\omega}$.

Now we plug in the Peter-Weyl decomposition $L^{2}(K) \cong \bigoplus_{\sigma \in \widehat{K}_{\omega}} \mathcal{H}_{\sigma}^{*} \otimes \mathcal{H}_{\sigma}$ of Lemma 2.2 (using Remark 2.3) together with the descriptions of $\lambda_{K}^{\bar{\omega}}$ and $\rho_{K}^{\omega}$ with respect to this decomposition to obtain the following.

Proposition 2.5. Let $V$ be an $\omega$-representation of a compact subgroup $K$ of $G$ on $\mathcal{H}$. Then there is a unitary

$$
\Phi: \mathcal{H} \otimes L^{2}(G) \rightarrow \bigoplus_{\sigma \in \widehat{K}_{\omega}} L^{2}\left(G \times_{K, V \otimes \sigma^{*}}\left(\mathcal{H} \otimes \mathcal{H}_{\sigma}^{*}\right)\right) \otimes \mathcal{H}_{\sigma}
$$

which intertwines the $\omega$-representations $\left.V \otimes \rho_{G}\right|_{K}$ of $K$ on $\mathcal{H} \otimes L^{2}(G)$ with the $\omega$-representation $\bigoplus_{\sigma \in \widehat{K}_{\omega}} 1_{L^{2}\left(G \times_{K} \mathcal{H}_{\sigma} \otimes \mathcal{H}_{\sigma}^{*}\right)} \otimes \sigma$. Moreover,

$$
\mathcal{K}\left(\mathcal{H} \otimes L^{2}(G)\right)^{K, \operatorname{Ad}\left(V \otimes \rho_{G}\right)} \cong \bigoplus_{\sigma \in \widehat{K}_{\omega}} \mathcal{K}\left(L^{2}\left(G \times_{K, V \otimes \sigma^{*}}\left(\mathcal{H} \otimes \mathcal{H}_{\sigma}^{*}\right)\right)\right) \otimes 1_{\mathcal{H}_{\sigma}} .
$$


Proof. The first assertion follows from the above discussion and the second from Remark 2.1.

Notation. Since the Hilbert spaces $L^{2}\left(G \times_{K, V \otimes \sigma^{*}}\left(\mathcal{H} \otimes \mathcal{H}_{\sigma}^{*}\right)\right)$ are going to be ubiquitous in the sequel, we are going to introduce the notation

$$
\mathcal{W}_{V, \sigma}:=L^{2}\left(G \times_{K, V \otimes \sigma^{*}}\left(\mathcal{H} \otimes \mathcal{H}_{\sigma}^{*}\right)\right)
$$

in an attempt to make some of our formulas easier to parse.

Remark 2.6. The above proposition shows that the fixed point algebra $\mathcal{K}(\mathcal{H} \otimes$ $\left.L^{2}(G)\right)^{K, \operatorname{Ad}\left(V \otimes \rho_{G}\right)}$ decomposes into blocks of compact operators such that each $\sigma \in \widehat{K}_{\omega}$ provides the block $\mathcal{K}\left(\mathcal{W}_{V, \sigma}\right)$ with multiplicity $d_{\sigma}:=\operatorname{dim} \mathcal{H}_{\sigma}$. Therefore, as a $C^{*}$-algebra, the fixed point algebra is isomorphic to $\bigoplus_{\sigma \in \widehat{K}_{\omega}} \mathcal{K}\left(\mathcal{W}_{V, \sigma}\right)$. Thus there is a bijection

$$
\operatorname{ind}_{K}: \widehat{K}_{\omega} \rightarrow\left(\mathcal{K}\left(\mathcal{H} \otimes L^{2}(G)\right)^{K, \operatorname{Ad}\left(V \otimes \rho_{G}\right)}\right)^{\wedge}
$$

which sends $\sigma \in \widehat{K}_{\omega}$ to the projection

$$
\operatorname{ind}_{K} \sigma: \mathcal{K}\left(\mathcal{H} \otimes L^{2}(G)\right)^{K, \operatorname{Ad}\left(V \otimes \rho_{G}\right)} \rightarrow \mathcal{K}\left(\mathcal{W}_{V, \sigma}\right) .
$$

Remark 2.7. By composing with the natural map of $U\left(\mathcal{H}_{\sigma}\right)$ onto $P U\left(\mathcal{H}_{\sigma}\right)$, each $\omega$ representation $\sigma$ determines a projective representation. If $\sigma^{\prime}$ is an $\omega^{\prime}$-representation representing the same projective representation, then $[\omega]=\left[\omega^{\prime}\right] \in H^{2}(K, \mathbb{T})$. Thus we can speak of the collection of equivalence classes $\widehat{K}_{[\omega]}$ of irreducible projective representations with class $[\omega] \in H^{2}(K, \mathbb{T})$. (Of course, there is an obvious bijection of $\widehat{K}_{\omega}$ with $\widehat{K}_{[\omega]}$.) Thus if $B:=\mathcal{K}(\mathcal{H})$, then an action $\beta: K \rightarrow$ Aut $B$ determines a class $[\omega] \in H^{2}(K, \mathbb{T})$ which does not depend on our choice of lift $V: K \rightarrow U(\mathcal{H})$. Therefore, the previous discussion says we have an isomorphism

$$
\left(B \otimes \mathcal{K}\left(L^{2}(G)\right)\right)^{\beta \otimes \operatorname{Ad} \rho_{G}} \cong \bigoplus_{\sigma \in \widehat{K}_{[\omega]}} \mathcal{K}_{\sigma}\left(\mathcal{W}_{V, \sigma}\right),
$$

for any choice of lift $V: K \rightarrow U(\mathcal{H})$ for $\beta$. Moreover, as a subalgebra of $\mathcal{K}(\mathcal{H} \otimes$ $\left.L^{2}(G)\right)$, each summand $\mathcal{K}_{\sigma}\left(\mathcal{W}_{V, \sigma}\right)$ appears with multiplicity $d_{\sigma}$.

In what follows, we call the summand $\mathcal{K}_{\sigma}\left(\mathcal{W}_{V, \sigma}\right)$ in (2.3) the summand of $(B \otimes$ $\left.\mathcal{K}\left(L^{2}(G)\right)\right)^{\beta \otimes \operatorname{Ad} \rho_{G}}$ of type $\sigma$.

As an immediate consequence of the above results (and conventions in Remark 2.6), we obtain the following.

Proposition 2.8. Suppose that $\beta: G \rightarrow$ Aut $B$ is a strictly proper action of $G$ on the continuous-trace $C^{*}$-algebra $B$ with spectrum $X$. Let $\left[\omega_{x}\right] \in H^{2}\left(G_{x}, \mathbb{T}\right)$ denote the Mackey obstruction of $\beta^{x}: G_{x} \rightarrow$ Aut $B(x)$. Then there is a canonical bijection

$$
\operatorname{ind}_{x}: \widehat{G}_{x,\left[\omega_{x}\right]} \rightarrow\left(\left(B(x) \otimes \mathcal{K}\left(L^{2}(G)\right)\right)^{G_{x}, \beta^{x} \otimes \operatorname{Ad} \rho_{G}}\right)^{\wedge} .
$$

Moreover, the diagram

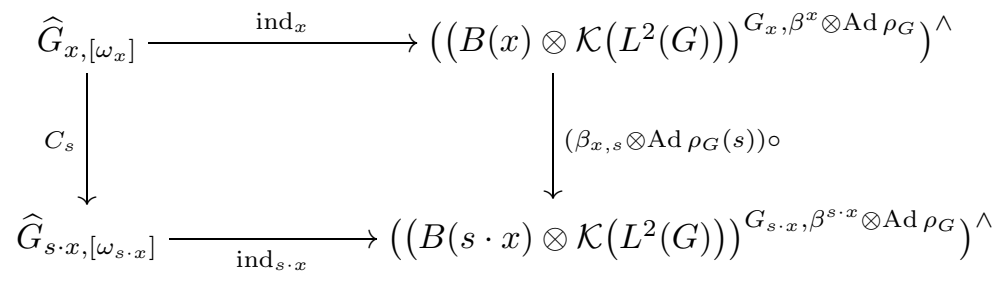


commutes, where $\beta_{x, s}: B(x) \rightarrow B(s \cdot x)$ is the isomorphism given in (1.1) and $C_{s}$ is given by $\sigma \mapsto s \cdot \sigma$ with $s \cdot \sigma(k)=\sigma\left(s^{-1} k s\right)$.

Proof. The only issue is the commutativity of the diagram. But (1.2) implies that conjugation of $\beta^{x} \otimes \operatorname{Ad} \rho_{G}$ by the isomorphism $\beta_{x, s} \otimes \operatorname{Ad} \rho_{G}(s)$ gives the action $\beta^{s \cdot x} \otimes \operatorname{Ad} \rho_{G}$. The rest follows from straightforward computations.

Recall that every irreducible representation of a $C_{0}(Y)$-algebra $A$ factors through a fibre, so that $\widehat{A}=\coprod_{y \in Y} \widehat{A}(y)$, Theorem 1.2 and Proposition 2.8 imply the following version of the Mackey-Green-Rieffel machine.

Theorem 2.9 (Mackey-Green-Rieffel). Suppose that $\beta: G \rightarrow$ Aut $B$ is a strictly proper action on the continuous-trace $C^{*}$-algebra with spectrum $X$. For each $x \in X$, let $\left[\omega_{x}\right] \in H^{2}\left(G_{x}, \mathbb{T}\right)$ be the Mackey obstruction for $\beta^{x}: G_{x} \rightarrow$ Aut $B(x)$ (where $\beta^{x}$ is the induced action of the stabilizer subgroup $G_{x}$ on the fibre $\left.B(x)\right)$. Let

$$
\operatorname{Stab}\left(X_{\beta}\right)^{\wedge}:=\left\{(x, \sigma): x \in X \text { and } \sigma \in \widehat{G}_{x,\left[\omega_{x}\right]}\right\}
$$

and let $G$ act on $\operatorname{Stab}\left(X_{\beta}\right)^{\wedge}$ via $s \cdot(x, \sigma):=(s \cdot x, s \cdot \sigma)$ with $s \cdot \sigma(k)=\sigma\left(s^{-1} k s\right)$. Then there is a surjective map

$$
\text { Ind : } \operatorname{Stab}\left(X_{\beta}\right)^{\wedge} \rightarrow\left(B \rtimes_{\beta} G\right)^{\wedge} \cong\left(\left(B \otimes \mathcal{K}\left(L^{2}(G)\right)\right)^{G, \beta \otimes \operatorname{Ad} \rho_{G}}\right)^{\wedge},
$$

given by sending $(x, \sigma) \in \operatorname{Stab}\left(X_{\beta}\right)^{\wedge}$ to the corresponding representation of the fibre $\left(B(x) \otimes \mathcal{K}\left(L^{2}(G)\right)\right)^{G_{x}, \beta^{x} \otimes \operatorname{Ad} \rho_{G}}$ (as in Proposition 2.8), which factors through a bijection of $G \backslash \operatorname{Stab}\left(X_{\beta}\right)^{\wedge}$ onto $\left(B \rtimes_{\beta} G\right)^{\wedge}$.

Remark 2.10. We should compare our version of the Mackey-Green-Rieffel machine with the classical approach. There we start with the irreducible representation $\pi_{x}: B \rightarrow \mathcal{K}\left(\mathcal{H}_{x}\right)$ (essentially evaluation at $x$ ), and let $V_{x}: G_{x} \rightarrow U\left(\mathcal{H}_{x}\right)$ and $\omega_{x} \in Z^{2}\left(G_{x}, \mathbb{T}\right)$ be as above. Then if $\sigma \in \widehat{G}_{x, \omega_{x}}$ we obtain an irreducible unitary representation $\left(\pi_{x} \otimes 1_{\mathcal{H}_{\sigma}^{*}}\right) \rtimes\left(V_{x} \otimes \sigma^{*}\right)$ of $B \rtimes_{\beta} G_{x}$ on $\mathcal{H}_{x} \otimes \mathcal{H}_{\sigma}^{*}$. Then we obtain an irreducible representation of $B \rtimes_{\beta} G$ via induction: $\operatorname{Ind}_{G_{x}}^{G}\left(\left(\pi_{x} \otimes 1_{\mathcal{H}_{\sigma}^{*}}\right) \rtimes\left(V_{x} \otimes \sigma^{*}\right)\right)$. Both this induced representation and the induced representations $\operatorname{ind}_{x}(\sigma)$ (defined in Proposition 2.8 and used in Theorem 2.9) act on $L^{2}\left(G \times_{G_{x}, V_{x} \otimes \sigma^{*}}\left(\mathcal{H}_{x} \otimes \mathcal{H}_{\sigma}^{*}\right)\right)=$ $\mathcal{W}_{V_{x}, \sigma}$. It is not difficult to check that the two representations are the same.

In order to understand the topology on $\left(B \rtimes_{\beta} G\right)^{\wedge}$, we will need to compare $\left(B(x) \otimes \mathcal{K}\left(L^{2}(G)\right)\right)^{G_{x}, \beta^{x} \otimes \operatorname{Ad} \rho_{G}}$ with the fixed point algebra $(B(x) \otimes$ $\left.\mathcal{K}\left(L^{2}(G)\right)\right)^{L, \beta^{x} \otimes \operatorname{Ad} \rho_{G}}$ for a closed subgroup $L$ of $G_{x}$. To simplify the notation, we consider the following setup. We suppose that $L$ is a closed subgroup of a compact subgroup $K$ of $G$ and $\omega \in Z^{2}(K, \mathbb{T})$. We let $V: K \rightarrow U(\mathcal{H})$ be an $\omega$-representation with $\beta:=\operatorname{Ad} V$. Then we may restrict everything to $L$ so that the fixed point algebra $\mathcal{K}\left(\mathcal{H} \otimes L^{2}(G)\right)^{K, \operatorname{Ad}\left(V \otimes \rho_{G}\right)}$ is a subset of $\mathcal{K}\left(\mathcal{H} \otimes L^{2}(G)\right)^{L, \operatorname{Ad}\left(V \otimes \rho_{G}\right)}$. Thus any block $\mathcal{K}\left(\mathcal{W}_{V, \sigma}\right)$ of type $\sigma \in \widehat{K}_{[\omega]}$ of the fixed point algebra for the $K$-action must be contained in a block $\mathcal{K}\left(L^{2}\left(G \times_{L, V \otimes \tau^{*}}\left(\mathcal{H} \times \mathcal{H}_{\tau}^{*}\right)\right)\right)=\mathcal{K}\left(\mathcal{W}_{V, \tau}\right)$ of type $\tau \in \widehat{L}_{[\omega]}$ in the fixed point algebra for the $L$-action. We aim to determine how many blocks of a given type $\sigma$ lie in a block of type $\tau$.

Towards this end, we note that Proposition 2.5 implies that we can decompose $\mathcal{H} \otimes L^{2}(G)$ as

$$
\bigoplus_{\sigma \in \widehat{K}_{\omega}} \mathcal{W}_{V, \sigma} \otimes \mathcal{H}_{\sigma}
$$


Furthermore, the decomposition in (2.4) is such that $V \otimes \rho_{G}$ is intertwined with the $\omega$-representation $\bigoplus_{\sigma} 1_{\mathcal{W}_{V, \sigma}} \otimes \sigma$. For each $\sigma \in \widehat{K}_{\sigma}$, we can decompose $\left.\sigma\right|_{L}$ into a direct sum $\bigoplus_{\tau \in \widehat{L}_{\omega}} m_{\tau}^{\sigma} \cdot \tau$ for appropriate multiplicities $m_{\tau}^{\sigma}$. Thus $\mathcal{H}_{\sigma}=$ $\bigoplus_{\tau \in \widehat{L}_{\omega}} \mathcal{H}_{m_{\tau}^{\sigma}} \otimes \mathcal{H}_{\tau}$, and (2.4) becomes

$$
\bigoplus_{\tau \in \widehat{L}_{\omega}}\left(\bigoplus_{\sigma \in \widehat{K}_{\omega}} \mathcal{W}_{V, \sigma} \otimes \mathcal{H}_{m_{\tau}^{\sigma}}\right) \otimes \mathcal{H}_{\tau}
$$

and the restriction of $V \otimes \rho_{G}$ to $L$ is given by

$$
\left.\left(V \otimes \rho_{G}\right)\right|_{L} \cong \bigoplus_{\tau \in \widehat{L}_{\omega}} 1_{\bigoplus_{\sigma \in \widehat{K}_{\omega}}} \mathcal{W}_{V, \sigma} \otimes \mathcal{H}_{m_{\tau}^{\sigma}} \otimes \tau
$$

This induces an isomorphism of the fixed point algebra

$$
\mathcal{K}\left(\mathcal{H} \otimes L^{2}(G)\right)^{L, \operatorname{Ad}\left(V \otimes \rho_{G}\right)} \cong \bigoplus_{\tau \in \widehat{L}_{\omega}} \mathcal{K}\left(\bigoplus_{\sigma \in \widehat{K}_{\omega}} \mathcal{W}_{V, \sigma} \otimes \mathcal{H}_{m_{\tau}^{\sigma}}\right) \otimes 1_{\mathcal{H}_{\tau}}
$$

Since the decomposition of $\left.\left(V \otimes \rho_{G}\right)\right|_{L}$ into isotopes $\mathcal{K}_{\tau}$ for the representations $\tau \in \widehat{L}_{\omega}$ is unique, we conclude that

$$
\mathcal{K}_{\tau}=\mathcal{K}\left(\mathcal{W}_{V, \tau}\right) \cong \mathcal{K}\left(\bigoplus_{\sigma \in \widehat{K}_{\omega}} \mathcal{W}_{V, \sigma} \otimes \mathcal{H}_{m_{\tau}^{\sigma}}\right)
$$

where as above, we have written $\mathcal{W}_{V, \tau}$ for $L^{2}\left(G \times_{L, V \otimes \tau^{*}}\left(\mathcal{H} \otimes \mathcal{H}_{\tau}^{*}\right)\right)$.

Summing up, we have the following proposition.

Proposition 2.11. In the above setting, each block of type $\sigma \in \widehat{K}_{\omega}$ in the decomposition of $\mathcal{K}\left(\mathcal{H} \otimes L^{2}(G)\right)^{K, \operatorname{Ad}\left(V \otimes \rho_{G}\right)}$ appears with multiplicity $m_{\tau}^{\sigma}$ in each block of type $\tau \in \widehat{L}_{\omega}$ in the decomposition of $\mathcal{K}\left(\mathcal{H} \otimes L^{2}(G)\right)^{L, \operatorname{Ad}\left(V \otimes \rho_{G}\right)}$, where $m_{\tau}^{\sigma}$ is the multiplicity of $\tau$ in $\left.\sigma\right|_{L}$.

We will also need to know how exterior equivalence affects our fixed point algebras. Recall that $\alpha, \beta: L \rightarrow$ Aut $A$ are called exterior equivalent if there is a strictly continuous map $u: L \rightarrow U(A)$ such that

$$
\alpha(l)=\operatorname{Ad}(u)(l) \circ \beta(l) \quad \text { and } \quad u(l k)=u(l) \beta_{l}(u(k)) \quad \text { for all } k, l \in L .
$$

In our case, $A=\mathcal{K}(\mathcal{H})$ and the map $u$ in (2.7) is a strongly continuous map into $U(\mathcal{H})$ such that $\alpha=\operatorname{Ad}(u V)$. Since $G$ is second countable, we can choose a Borel cross-section $c: G / L \rightarrow G$ and decompose $L^{2}(G) \cong L^{2}(G / L) \otimes L^{2}(L)$ by sending $\xi \in L^{2}(G)$ to the element $\tilde{\xi} \in L^{2}(G / L) \otimes L^{2}(L) \cong L^{2}(G / L \times L)$ given by $\tilde{\xi}(s L, l)=\xi(c(s L) l)$. Then we obtain a decomposition

$$
\mathcal{H} \otimes L^{2}(G) \cong L^{2}(G / L) \otimes \mathcal{H} \otimes L^{2}(L)
$$

which intertwines the representation $\left.\left(V \otimes \rho_{G}\right)\right|_{L}$ with the representation $1_{L^{2}(G / L)} \otimes$ $\left(V \otimes \rho_{L}\right)$. Then it follows from Lemma 2.4 (applied twice), after identifying $\mathcal{H} \otimes$ $L^{2}(L)$ with $L^{2}(L, \mathcal{H})$, that the unitary $W: L^{2}(L, \mathcal{H}) \rightarrow L^{2}(L, \mathcal{H})$ given by

$$
(W \xi)(l):=V(l)^{*} u(l)^{*} V(l) \xi(l)
$$

intertwines $V \otimes \rho_{L}$ and $u V \otimes \rho_{L}$. Therefore, we get the following result.

Lemma 2.12. Let $W$ be as above and let $\tilde{W} \in U\left(\mathcal{H} \otimes L^{2}(G)\right)$ corresponding to $1_{L^{2}(G / L)} \otimes W$ under the decomposition $\mathcal{H} \otimes L^{2}(G) \cong \mathcal{H} \otimes L^{2}(G / L) \otimes L^{2}(L)$. Then

$$
\mathcal{K}\left(\mathcal{H} \otimes L^{2}(G)\right)^{L, \operatorname{Ad}\left(u V \otimes \rho_{G}\right)} \cong \tilde{W}\left(\mathcal{K}\left(\mathcal{H} \otimes L^{2}(G)\right)^{L, \operatorname{Ad}\left(V \otimes \rho_{G}\right)}\right) \tilde{W}^{*} .
$$




\section{The $\operatorname{SPACE} \operatorname{Stab}\left(X_{\beta}\right)^{\wedge}$}

Our object in this section is to equip $\operatorname{Stab}\left(X_{\beta}\right)^{\wedge}$ with a natural topology so that the induction map Ind $: \operatorname{Stab}\left(X_{\beta}\right)^{\wedge} \rightarrow\left(B \rtimes_{\beta} G\right)^{\wedge}$ of Theorem 2.9 induces a homeomorphism of the quotient topological space $G \backslash \operatorname{Stab}\left(X_{\beta}\right)^{\wedge}$ onto $\left(B \rtimes_{\beta} G\right)^{\wedge}$ for any strictly proper action $\beta$ of $G$ on a continuous-trace $C^{*}$-algebra $B$ with spectrum $X$.

First, we need some general observations. Let $A$ be an $X \rtimes G$-algebra with respect to $\alpha: G \rightarrow$ Aut $A$ so that we can form the generalized fixed point algebra $A^{G, \alpha}$, and recall that $A^{G, \alpha}$ is a $C_{0}(G \backslash X)$-algebra. If $q: X \rightarrow G \backslash X$ is the orbit map, we can form the pull-back

$$
q^{*} A^{G, \alpha}=C_{0}(X) \otimes_{C_{0}(G \backslash X)} A^{G, \alpha} .
$$

Using the description of the primitive ideal space from [24, Lemma 1.1], it is easy to identify the fibre $q^{*} A^{G, \alpha}(x)$ with $A^{G, \alpha}(G \cdot x)$. Recall that Lemma 1.1 implies that $A^{G, \alpha}(G \cdot x)$ is isomorphic to $A(x)^{G_{x}, \alpha^{x}}$ via evaluation at $x$.

We let

$$
A_{\text {fix }}:=\left\{a \in A=\Gamma_{0}(X, \mathscr{A}): a(x) \in A(x)^{G_{x}, \alpha^{x}} \text { for all } x\right\} .
$$

Then $A_{\text {fix }}$ is a $C_{0}(X)$-subalgebra of $A$. In fact it is $G$-invariant. To see this, let $a \in \mathscr{A}_{\mathrm{fix}}$ and $b:=\alpha_{s}(a)$ for some $s \in G$. Let $k \in G_{x}$. Appealing to (1.1) and (1.2) as necessary, we have

$$
\begin{aligned}
\alpha_{k}^{x}(b(x)) & =\alpha_{x, k}(b(x))=\alpha_{k s}(a)(x) \\
& =\alpha_{s^{-1} \cdot x, k s}\left(a\left(s^{-1} \cdot x\right)\right) \\
& =\alpha_{s^{-1} \cdot x, s} \circ \alpha_{s^{-1} \cdot x, s^{-1} k s}\left(a\left(s^{-1} \cdot x\right)\right)
\end{aligned}
$$

which, since $s^{-1} k s \in G_{s^{-1} \cdot x}, \alpha_{s^{-1} \cdot x, s^{-1} k s}=\alpha_{s^{-1} k s}^{s^{-1} \cdot x}$ and $a \in A_{\mathrm{fix}}$, is

$$
\begin{aligned}
& =\alpha_{s^{-1} \cdot x, s}\left(a\left(s^{-1} \cdot x\right)\right) \\
& =\alpha_{s}(a)(x)=b(x) .
\end{aligned}
$$

Lemma 3.1. Viewing $A^{G, \alpha} \subseteq \Gamma_{b}(X, \mathscr{A})$ as in Lemma 1.1, it follows that if $\phi \in$ $C_{0}(X)$ and $a \in A^{G, \alpha}$, then $\phi \cdot a \in A_{\text {fix }}($ where $(\phi \cdot a)(x)=\phi(x) a(x))$. In particular, $A_{\text {fix }}$ is a $C_{0}(X)$-algebra with fibres $A_{\text {fix }}(x) \cong A(x)^{G_{x}, \alpha^{x}}$ via evaluation at $x$. Its spectrum can be identified with the set $\widehat{A}_{\mathrm{fix}}=\left\{(x, \pi): x \in X\right.$ and $\left.\pi \in\left(A(x)^{G_{x}, \alpha^{x}}\right)^{\wedge}\right\}$. Furthermore, the induced $G$-action on $\widehat{A}_{\text {fix }}$ is given by $s \cdot(x, \pi)=\left(s \cdot x, \pi \circ \alpha_{s^{-1} \cdot x, s}\right)$.

Proof. The first assertion is straightforward, as any $a \in A^{G, \alpha}$ must satisfy $a(x) \in$ $A(x)^{G_{x}, \alpha^{x}}$. But evaluation at $x$ clearly defines an injection of $A_{\text {fix }}(x)$ into $A(x)^{G_{x}, \alpha^{x}}$. But if $a_{0} \in A(x)^{G_{x}, \alpha^{x}}$, then Lemma 1.1 implies that there is a $a \in A^{G, \alpha}$ such that $a(x)=a_{0}$. We let $\phi \in C_{0}(X)$ be such that $\phi(x)=1$. Then $\phi \cdot a \in A_{\text {fix }}$ and $(\phi \cdot a)(x)=a_{0}$. Hence $A_{\text {fix }}$ is a $C_{0}(X)$-algebra with fibres, as claimed. The remaining assertions are straightforward consequences of the fact that the spectrum of a $C_{0}(X)$-algebra is the disjoint union of the spectrums of its fibres.

Proposition 3.2. Let $A$ be an $X \rtimes G$-algebra as above. Then the map $\phi \otimes a \mapsto \phi \cdot a$ induces a $C_{0}(X)$-isomorphism of the pull-back $q^{*} A^{G, \alpha}$ onto $A_{\mathrm{fix}}$. 
Proof. In view of Lemma 3.1 it is clear that $\phi \otimes a \mapsto \phi \cdot a$ induces a $C_{0}(X)$ homomorphism which is an isomorphism on the fibres. Hence it is an isomorphism, as claimed.

By Lemma 1.1, $A^{G, \alpha}$ is a $C_{0}(G \backslash X)$-algebra with evaluation at $x$ inducing an isomorphism of the fibres $A^{G, \alpha}(G \cdot x)$ with $A(x)^{G_{x}, \alpha^{x}}$. Hence the irreducible representations of $A^{G, \alpha}$ are given by pairs $[x, \pi]$ with $x \in X$ and $\pi \in\left(A^{G_{x}, \alpha^{x}}\right)^{\wedge}$ so that $[x, \pi](a)=\pi(a(x))$. Furthermore $[x, \pi]=[y, \rho]$ exactly when $(y, \rho)=(s \cdot x, \pi \circ$ $\left.\alpha_{s^{-1} \cdot x, s}\right)$. Combining this with Lemma 3.1, we see that the map $(x, \pi) \mapsto[x, \pi]$ induces a bijection of $G \backslash \widehat{A}_{\text {fix }}$ onto $\left(A^{G, \alpha}\right)^{\wedge}$.

Proposition 3.3. Let $A$ be an $X \rtimes G$-algebra as above. Then the map $(x, \pi) \mapsto$ $[x, \pi]$ induces a homeomorphism of $G \backslash \widehat{A}_{\text {fix }}$ and $\left(A^{G, \alpha}\right)^{\wedge}$.

Proof. There is a natural homomorphism of $A^{G, \alpha}$ into the multiplier algebra $M\left(A_{\text {fix }}\right)$ and $[x, \pi]$ is just the restriction of the natural extension of $(x, \pi)$ to $M\left(A_{\mathrm{fix}}\right)$. Hence $(x, \pi) \mapsto[x, \pi]$ is continuous by general nonsense (see [12, Proposition 9]).

Thus it will suffice to see that the map is open. For this, it suffices to show that if $\left[x_{i}, \pi_{i}\right] \rightarrow[x, \pi]$, then we can pass to a subsequence, relabel, and find $\left(y_{i}, \rho_{i}\right) \rightarrow$ $(x, \pi)$ such that $\left[y_{i}, \rho_{i}\right]=\left[x_{i}, \pi_{i}\right]$ (see [30, Proposition 1.15]). However, since $A^{G, \alpha}$ is a $C_{0}(G \backslash X)$-algebra, we must have $G \cdot x_{i} \rightarrow G \cdot x$, and after passing to a subsequence, relabeling and adjusting the $\pi_{i}$ as necessary, we can assume that $x_{i} \rightarrow x$. But [24, Lemma 1.1] implies that

$$
\left(q^{*} A^{G, \alpha}\right)^{\wedge}=\left\{(x,[y, \rho]) \in X \times\left(A^{G, \alpha}\right)^{\wedge}: y \in G \cdot x\right\}
$$

has the relative product topology. Hence $\left(x_{i},\left[x_{i}, \pi_{i}\right]\right) \rightarrow(x,[x, \pi])$ in $\left(q^{*} A^{G, \alpha}\right)^{\wedge}$. But the isomorphism of $q^{*} A^{G, \alpha}$ with $A_{\text {fix }}$ given in Proposition 3.2 intertwines $(x,[x, \pi])$ with $(x, \pi)$. Hence we must have $\left(x_{i}, \pi_{i}\right) \rightarrow(x, \pi)$ in $\widehat{A}_{\text {fix }}$.

We want to apply the previous discussion to $(A, \alpha)=\left(B \otimes \mathcal{K}\left(L^{2}(G)\right), \beta \otimes \operatorname{Ad} \rho_{G}\right)$. Proposition 2.8 implies that the map $(x, \sigma) \mapsto\left(x, \operatorname{ind}_{x} \sigma\right)$ is a $G$-equivariant map of $\operatorname{Stab}\left(X_{\beta}\right)^{\wedge}$ onto $\left(\left(B \otimes \mathcal{K}\left(L^{2}(G)\right)\right)_{\text {fix }}\right)^{\wedge}$. This gives us a natural choice of a topology for $\operatorname{Stab}\left(X_{\beta}\right)^{\wedge}$.

Definition 3.4. We equip $\operatorname{Stab}\left(X_{\beta}\right)^{\wedge}$ with the topology making its identification with $\left(\left(B \otimes \mathcal{K}\left(L^{2}(G)\right)\right)_{\text {fix }}\right)^{\wedge}$ a homeomorphism.

Having made this definition, our desired description of the spectrum of $B \rtimes_{\beta} G$ follows immediately from Proposition 3.3 and Theorem 1.2

Theorem 3.5. Suppose that B is a strictly proper continuous-trace $C^{*}$-algebra with spectrum $X$. Then the map

$$
\text { Ind : } \operatorname{Stab}\left(X_{\beta}\right)^{\wedge} \rightarrow\left(B \rtimes_{\beta} G\right)^{\wedge}
$$

is continuous and open, and hence factors through a homeomorphism of $G \backslash \operatorname{Stab}\left(X_{\beta}\right)^{\wedge}$ with $\left(B \rtimes_{\beta} G\right)^{\wedge}$.

\section{Actions of Countable groups and Lie Groups}

Our Theorem 3.5 reduces the problem of understanding the topology on the spectrum of $B \rtimes_{\beta} G$ to understanding the topology of $\operatorname{Stab}\left(X_{\beta}\right)^{\wedge}$. In this section we want to give a nice description of this topology, first in the case that $G$ is discrete, 
and then in the case $X=\widehat{B}$ is a manifold and $G$ is a Lie group acting smoothly on $X$.

Before stating our results, we need to recall some common terminology regarding projective representations and the corresponding $\omega$-representations. First, if $\tau$ is an $\omega^{\prime}$-representation and $\sigma$ is an $\omega$-representation of the group $K$, then we say $\tau$ is a subrepresentation of $\sigma$ (written $\tau \leq \sigma)$ if $[\omega]=\left[\omega^{\prime}\right]$ in $H^{2}(K, \mathbb{T}$ ), and if $f: G \rightarrow \mathbb{T}$ is a Borel map such that $\omega=\delta f \cdot \omega^{\prime}$ (where $\delta$ is the boundary map), then the $\omega$-representation $\tau^{\prime}:=f \cdot \tau$ is unitarily equivalent to a subrepresentation of $\sigma$. Note also that if $L$ is a closed subgroup of $K$ and if $\omega \in Z^{2}(K, \mathbb{T})$, then the class of $\left.\omega\right|_{L}$ in $H^{2}(L, \mathbb{T})$ depends only on the class of $\omega$ in $H^{2}(K, \mathbb{T})$.

Our main results are as follows.

Theorem 4.1. Suppose that $\beta: G \rightarrow$ Aut $B$ is a strictly proper action of a countable group $G$ on a separable continuous-trace $C^{*}$-algebra $B$ with spectrum $X$. Then $\left(x_{n}, \sigma_{n}\right) \rightarrow(x, \sigma)$ in $\operatorname{Stab}\left(X_{\beta}\right)^{\wedge}$ if and only if

(a) $x_{n} \rightarrow x$ in $X$ and

(b) there is a $N \in \mathbb{N}$ such that for all $n \geq N$ we have $G_{x_{n}} \subseteq G_{x}$ and $\sigma_{n} \leq\left.\sigma\right|_{G_{x_{n}}}$.

We say that a Lie group action $\beta: G \rightarrow$ Aut $B$ on a $C^{*}$-algebra $B$ with spectrum $X$ is differentiable if $X$ is a manifold and each $s \in G$ acts by a diffeomorphism on $X$.

Theorem 4.2. Suppose that $\beta: G \rightarrow$ Aut $B$ is a strictly proper differentiable action of a Lie group $G$ on a separable continuous-trace $C^{*}$-algebra $B$. Then $\left(x_{n}, \sigma_{n}\right) \rightarrow$ $(x, \sigma)$ if and only if

(a) $x_{n} \rightarrow x$ in $X$ and

(b) each subsequence of $\left\{\left(x_{n}, \sigma_{n}\right)\right\}$ has a subsequence $\left\{\left(y_{l}, \rho_{l}\right)\right\}$ such that there is a sequence $\left\{s_{l}\right\}$ in $G$ such that $s_{l} \rightarrow e$ in $G$ and a closed subgroup $L \subseteq G_{x}$ such that for all $l$,

(i) $G_{s_{l} \cdot y_{l}}=s_{l} G_{y_{l}} s_{l}^{-1}=L$ and

(ii) $s_{l} \cdot \rho_{l} \leq\left.\sigma\right|_{L}$.

First some preliminary observations. Recall that if $D$ is a $C^{*}$-subalgebra of $\mathcal{K}(\mathcal{H})$, then $D \cong \bigoplus_{\tau \in \widehat{D}} \mathcal{K}\left(\mathcal{H}_{\tau}\right)$. Let $p_{\tau}$ be the projection in $M(D)$ corresponding to $\mathcal{K}\left(\mathcal{H}_{\tau}\right)$. If $C$ is a $C^{*}$-subalgebra of $D$, then $\left.\tau\right|_{C}$ maps $C$ onto $C_{\tau}:=p_{\tau} C p_{\tau} \subseteq \mathcal{K}\left(\mathcal{H}_{\tau}\right)$. If we decompose $C_{\tau} \cong \bigoplus_{\sigma \in \widehat{C}_{\tau}} \mathcal{K}\left(\mathcal{H}_{\sigma}\right)$, then $\widehat{C}_{\tau}$ can be identified with those $\sigma \in \widehat{C}$ which appear as subrepresentations of $\left.\tau\right|_{C}$; that is, $\widehat{C}_{\tau}=\left\{\sigma \in \widehat{C}: \sigma \leq\left.\tau\right|_{C}\right\}$. We want to apply these observations to the following situation.

Example 4.3. Let $L$ be a closed subgroup of a compact subgroup $K$ of $G$. Suppose that $V: K \rightarrow U(\mathcal{H})$ is an $\omega$-representation. Then

$$
D:=\left(\mathcal{K}\left(\mathcal{H} \otimes L^{2}(G)\right)\right)^{L, \operatorname{Ad}\left(V \otimes \rho_{G}\right)}
$$

is a subalgebra of $\mathcal{K}\left(\mathcal{H} \otimes L^{2}(G)\right)$ which contains the fixed point algebra

$$
C:=\left(\mathcal{K}\left(\mathcal{H} \otimes L^{2}(G)\right)\right)^{K, \operatorname{Ad}\left(V \otimes \rho_{G}\right)} .
$$

Thus if we let $\operatorname{ind}_{K}: \widehat{K}_{\omega} \rightarrow \widehat{C}$ and $\operatorname{ind}_{L}: \widehat{L}_{\omega} \rightarrow \widehat{D}$ be the bijections from Remark 2.6, then after combining the above considerations with Proposition 2.11 we see that

$$
\operatorname{ind}_{K} \sigma \leq\left.\left(\operatorname{ind}_{L} \tau\right)\right|_{C} \Longleftrightarrow \tau \leq\left.\sigma\right|_{L} .
$$


Lemma 4.4. Let $\mathbb{N}_{\infty}$ be the one-point compactification of $\mathbb{N}$ and let $C \subseteq D \subseteq \mathcal{K}(\mathcal{H})$ be $C^{*}$-subalgebras. Let

$$
A:=\left\{f \in C\left(\mathbb{N}_{\infty}, D\right): f(\infty) \in C\right\} .
$$

Then $\widehat{A}=(\mathbb{N} \times \widehat{D}) \amalg \widehat{C}$ and a sequence $\left\{\left(n, \rho_{n}\right)\right\}$ in $\mathbb{N} \times \widehat{D}$ converges to $\sigma \in \widehat{C}$ if and only if there is an $N \in \mathbb{N}$ such that $\sigma \leq\left.\rho_{n}\right|_{C}$ for all $n \geq N$.

Proof. Assume that $\left(n, \rho_{n}\right) \rightarrow \sigma$ in $\widehat{A}$. If the assertion in the lemma is false, then we can pass to a subsequence, relabel, and assume that for all $n, \sigma \not \leq\left.\rho_{n}\right|_{C}$.

Let $B=C\left(\mathbb{N}_{\infty}, C\right)$ viewed as a subalgebra of $A$. Since restriction gives a continuous map from $\operatorname{Rep}(A) \rightarrow \operatorname{Rep}(B)$ in the Fell topology (see [6, §1.2]), we have $\left.\left(n, \rho_{n}\right)\right|_{C} \rightarrow \sigma$ in $\operatorname{Rep}(B)$. Then, identifying $C$ with the constant functions in $C\left(\mathbb{N}_{\infty}, C\right)$, we see that $\left.\rho_{n}\right|_{C} \rightarrow \sigma$ in $\operatorname{Rep}(C)$. Since $\left.\rho_{n}\right|_{C}$ decomposes as a direct sum of irreducibles, 29, Theorem 2.2] implies that, after passing to a subsequence and relabeling, we can find irreducible subrepresentations $\sigma_{n} \leq\left.\rho_{n}\right|_{C}$ such that $\sigma_{n} \rightarrow \sigma$ in $\widehat{C}$. Since $\widehat{C}$ is clearly discrete, we eventually have $\sigma_{n}=\sigma$, which is a contradiction.

Conversely, assume that $\sigma \leq\left.\rho_{n}\right|_{C}$ for all $n \geq N$. Let $a \in C\left(\mathbb{N}_{\infty}, D\right)$ be such that $\left(n, \rho_{n}\right)(a)=\rho_{n}(a(n))=0$ for all $n \in \mathbb{N}$. We want to see that $a(\infty)=0$. Let $\tilde{a} \in A$ be the constant function with value $a(\infty)$. Then $\|a(n)-\tilde{a}(n)\| \rightarrow 0$ as $n \rightarrow \infty$. Since $\sigma$ is a subrepresentation of $\left.\rho_{n}\right|_{C}$, this implies that

$$
\|\sigma(a(\infty))\| \leq\left\|\rho_{n}(a(\infty))\right\|=\left\|\rho_{n}(a(n)-\tilde{a}(n))\right\| \leq\|a(n)-\tilde{a}(n)\|
$$

for any $n \geq N$. Hence $\sigma(a(\infty))=0$. Since we can apply this to any subsequence of $\left\{\left(n, \rho_{n}\right)\right\}$, it follows that $\left(n, \rho_{n}\right) \rightarrow \sigma$, as claimed.

Lemma 4.5. Suppose that $\beta: G \rightarrow$ Aut $B$ is a strictly proper action of a second countable locally compact group on a separable, stable continuous-trace $C^{*}$-algebra $B$ with spectrum $X$. Assume that $\left\{x_{n}\right\}$ is a sequence in $X$ converging to $x$ such that

(a) for all $n \neq m, x_{n} \neq x_{m} \neq x$ and

(b) there is a fixed subgroup $L \subseteq G_{x}:=K$ such that $G_{x_{n}}=L$ for all $n$.

Moreover, let $\pi: B \rightarrow \mathcal{K}(\mathcal{H})$ be an irreducible representation of $B$ corresponding to $x$ and let $V: K \rightarrow U(\mathcal{H})$ be an $\omega$-representation implementing the action of $K$ on $B(x) \cong \mathcal{K}(\mathcal{H})$. Let

$$
C:=\left(\mathcal{K}\left(\mathcal{H} \otimes L^{2}(G)\right)\right)^{K, \operatorname{Ad}\left(V \otimes \rho_{G}\right)} \quad \text { and } \quad D=\left(\mathcal{K}\left(\mathcal{H} \otimes L^{2}(G)\right)\right)^{L, \operatorname{Ad}\left(V \otimes \rho_{G}\right)} .
$$

Then there exists $N \in \mathbb{N}$ such that, after identifying $S=\left\{x_{n}: n \geq N\right\} \cup\{x\}$ with $\mathbb{N}_{\infty}$, we get an isomorphism of

$$
\left(B \otimes \mathcal{K}\left(L^{2}(G)\right)\right)_{\mathrm{fix}, S} \quad \text { with } \quad\left\{a \in C\left(\mathbb{N}_{\infty}, D\right): a(\infty) \in C\right\},
$$

where $\left(B \otimes \mathcal{K}\left(L^{2}(G)\right)\right)_{\mathrm{fix}, S}$ is the restriction of the $C_{0}(X)$-algebra $\left(B \otimes \mathcal{K}\left(L^{2}(G)\right)\right)_{\mathrm{fix}}$ to the closed subset $S$ of $X$.

Proof. Let $B_{S}$ be the restriction of $B$ to $S \cong \mathbb{N}_{\infty}$. Since $B$ is a stable continuoustrace $C^{*}$-algebra, $B_{S} \cong C\left(\mathbb{N}_{\infty}, \mathcal{K}(\mathcal{H})\right)$ and the isomorphism transports the actions $\beta^{x_{n}}$ of $L=G_{x_{n}}$ on $B\left(x_{n}\right)$ to appropriate actions $\beta^{n}$ of $L$ on $\mathcal{K}(\mathcal{H})$. Also let $\beta^{\infty}$ be the restriction of the given $K$-action $\beta^{x}$ on $\mathcal{K}(\mathcal{H})$ to $L$. We obtain a $C\left(\mathbb{N}_{\infty}\right)$ linear action $\beta^{L}$ of $L$ on $C\left(\mathbb{N}_{\infty}, \mathcal{K}(\mathcal{H})\right)$ by $\beta_{l}^{L}(a)(n)=\beta^{n}(a(n))$. Since every $\widehat{L}_{\mathrm{ab}^{-}}$ bundle over $\mathbb{N}_{\infty}$ is trivial, it follows from [11, Theorem 5.4] that $\beta^{L}$ is classified 
up to exterior equivalence by the continuous Mackey-obstruction map $y \mapsto\left[\omega_{y}\right] \in$ $H^{2}(L, \mathbb{T})$ on $\mathbb{N}_{\infty}$. Since $L$ is compact, $H^{2}(L, \mathbb{T})$ is discrete (combine [16. Corollary 1] with the results at the beginning of [17, Chapter III] which imply that $H^{2}(L, \mathbb{T})$ is a countable, locally compact Hausdorff group, hence discrete). Thus we can assume that we have taken $N$ large enough so that $\left[\omega_{y}\right]=\left[\left.\omega\right|_{L}\right]$ for all $y$ (where $\omega$ is as in the statement of the lemma). It follows from [11, Theorem 5.4] that $\beta^{L}$ is exterior equivalent to the action defined by the constant field $\alpha=\left.\operatorname{id}_{C\left(\mathbb{N}_{\infty}\right)} \otimes \operatorname{Ad} V\right|_{L}$. Thus there is a continuous map $u: L \times \mathbb{N}_{\infty} \rightarrow U(\mathcal{H})$ such that for all $n \in \mathbb{N}_{\infty}$, $\beta_{l}^{n}=\operatorname{Ad}\left(u(l, y) \cdot V_{l}\right)$ and

$$
u(l k, y)=u(l, y) V_{l} u(k, y) V_{l}^{*} \quad \text { for all } a \in \mathcal{K}(\mathcal{H}), y \in \mathbb{N}_{\infty} \text { and } l, k \in L .
$$

Since $\beta^{\infty}=\operatorname{Ad} V=\operatorname{Ad}(u(\cdot, \infty) V)$, it follows that $u(l, \infty) \in \mathbb{T} I_{\mathcal{H}}$ for all $l$. Then (4.1) implies that $l \mapsto u(l, \infty)$ is a character. Multiplying each $u(\cdot, y)$ by the inverse of this character allows us to assume that $u(l, \infty)=1$ for all $l$.

After identifying $S$ with $\mathbb{N}_{\infty}$, we have, by definition,

$$
\begin{aligned}
& \left(B \otimes \mathcal{K}\left(L^{2}(G)\right)\right)_{\text {fix }, S} \cong\left\{a \in C\left(\mathbb{N}_{\infty}, \mathcal{K}\left(\mathcal{H} \otimes L^{2}(G)\right)\right):\right. \\
& \left.\qquad a(y) \in\left(\mathcal{K}\left(\mathcal{H} \otimes L^{2}(G)\right)\right)^{G_{y}, \operatorname{Ad}\left(u(\cdot y) V \otimes \rho_{G}\right)} \text { for all } y \in \mathbb{N}_{\infty}\right\},
\end{aligned}
$$

with $G_{y}=L$ if $y \in \mathbb{N}$ and $G_{\infty}=K$.

For each $n \in \mathbb{N}$, let $W_{n}: L^{2}(G, \mathcal{H}) \rightarrow L^{2}(G, \mathcal{H})$ be the unitary from Lemma 2.12 corresponding to the cocycle $l \mapsto u(l, n)$ from $L$ into $U(\mathcal{H})$. It then follows - see (2.8) and recall that $u$ is strongly continuous with $u(l, \infty)=1$ for all $l$ - that the sequence $\left\{W_{n}\right\}$ converges strongly to 1 . Thus, if we define $W_{\infty}$ to be 1 , we get an isomorphism $\Phi: C\left(N_{\infty}, \mathcal{K}\left(\mathcal{H} \otimes L^{2}(G)\right)\right) \rightarrow C\left(\mathbb{N}_{\infty}, \mathcal{K}\left(\mathcal{H} \otimes L^{2}(G)\right)\right)$ given by

$$
\Phi(a)(y)=W_{y} a(y) W_{y} \quad \text { for all } y \in \mathbb{N}_{\infty} .
$$

Lemma 2.12 implies that $\Phi$ maps $\left(B \otimes \mathcal{K}\left(L^{2}(G)\right)\right)_{\text {fix }, S}$ onto

$$
\begin{aligned}
& \left\{a \in C\left(\mathbb{N}_{\infty}, \mathcal{K}\left(\mathcal{H} \otimes L^{2}(G)\right)\right):\right. \\
& \left.\qquad a(y) \in\left(\mathcal{K}\left(\mathcal{H} \otimes L^{2}(G)\right)\right)^{G_{y}, \operatorname{Ad}\left(V \otimes \rho_{G}\right)} \text { for all } y \in \mathbb{N}_{\infty}\right\},
\end{aligned}
$$

which in the notation of the lemma, is exactly $\left\{a \in C\left(\mathbb{N}_{\infty}, D\right): a(\infty) \in C\right\}$.

Proof of Theorem 4.1. If $\beta$ is a strictly proper action on $B$, then $\beta \otimes$ id is a strictly proper action on $B \otimes \mathcal{K}(\mathcal{H})$. Furthermore, the corresponding "fix" algebras, $(B \otimes$ $\left.\mathcal{K}\left(L^{2}(G)\right)\right)_{\text {fix }}$ and $\left(B \otimes \mathcal{K}\left(\mathcal{H} \otimes L^{2}(G)\right)\right)_{\text {fix }}$ are Morita equivalent in such a way that the identification of the spectrum with $\operatorname{Stab}\left(X_{\beta}\right)^{\wedge}$ is preserved. Thus we may as well assume from the onset that $B$ is stable (so that we can apply Lemma 4.5 when appropriate).

Now suppose that $\left(x_{n}, \sigma_{n}\right) \rightarrow(x, \sigma)$ in $\operatorname{Stab}\left(X_{\beta}\right)^{\wedge}$. Since $\left(B \otimes \mathcal{K}\left(L^{2}(G)\right)\right)_{\text {fix }}$ is a $C_{0}(X)$-algebra, we must have $x_{n} \rightarrow x$ in $X$. Since $G$ is discrete, the action of $G$ on $X$ satisfies Palais's slice property (see Remark 4.12 and [20, Case 3 of Proposition 2.3.1]). Hence we may assume that there is an open neighborhood $U$ of $x$ such that $G_{y} \subseteq G_{x}$ for all $y \in U$. Thus there is an $N$ such that $n \geq N$ implies that $G_{x_{n}} \subseteq G_{x}$. Hence it will suffice to assume that $x_{n} \rightarrow x, G_{x_{n}} \subseteq G_{x}$ for all $n$ and show that the failure of condition (b) in the statement of the theorem results in a contradiction.

Since $G_{x}$ must be a finite group, it can have only finitely many subgroups. Thus we can pass to a subsequence and assume that there is a subgroup $L$ of $K:=G_{x}$ 
such that $G_{x_{n}}=L$ for all $n$ and such that condition (b) fails for this sequence. Let $S=\left\{x_{n}: n \geq 1\right\} \cup\{x\}$. As in the proof of Lemma 4.5, we obtain a $C(S)$-linear action of $L$ on $C(S, \mathcal{K}(\mathcal{H}))$. The Mackey obstruction at $x$ for this action is given by the restriction of the Mackey obstruction $\left[\omega_{x}\right]$ of $K$ to $L$. Since $x_{n} \mapsto\left[\omega_{x_{n}}\right]$ is continuous [11, Lemma 5.3], we have $\left[\omega_{x_{n}}\right] \rightarrow\left[\left.\omega\right|_{L}\right]$ in $H^{2}(L, \mathbb{T})$. But as $L$ is finite, $H^{2}(L, \mathbb{T})$ is finite and there is an $N$ such that $n \geq N$ implies that $\left[\omega_{x_{n}}\right]=\left[\left.\omega_{x}\right|_{L}\right]$. We'll assume that this holds for all $n$. Since we're assuming condition (b) fails, there is no $N$ such that $\sigma_{n} \leq\left.\sigma\right|_{L}$ for all $n \geq N$. Passing to a subsequence, we can assume that $\sigma_{n} \not \leq\left.\sigma\right|_{L}$ for all $n$. The sequence $\left\{x_{n}\right\}$ is either eventually constant or we can pass to a subsequence which, after relabeling, satisfies $x_{n} \neq x_{m} \neq x$ for all $n \neq m$. In the eventually constant case, we have $\sigma_{n} \rightarrow \sigma$ in the discrete set $\widehat{K}_{\omega} \cong\left(\mathcal{K}\left(\mathcal{H} \otimes L^{2}(G)\right)\right)^{K, \beta \otimes \operatorname{Ad} \rho^{\wedge}}$. But this implies that we eventually have $\sigma_{n}=\sigma$ which contradicts $\sigma_{n} \not \leq \sigma$. Otherwise we are in the situation of Lemma 4.5] and we can assume that $\left(n, \sigma_{n}\right) \rightarrow(\infty, \sigma)$ in $\left\{a \in C\left(\mathbb{N}_{\infty}, D\right): a(\infty) \in C\right\}$ with $C$ and $D$ as in Lemma 4.5. Then a combination of Lemma 4.4 and Example 4.3 gives a contradiction. Thus we have proved the forward implication in the theorem.

To prove the converse, let $\left(x_{n}, \sigma_{n}\right)$ and $(x, \sigma)$ satisfy conditions (a) and (b) in the theorem. It suffices to show that every subsequence of $\left\{\left(x_{n}, \sigma_{n}\right)\right\}$ has a subsequence converging to $(x, \sigma)$. Since every subsequence will still satisfy (a) and (b), it will suffice to see that the given sequence has a subsequence converging to $(x, \sigma)$. But either $\left\{x_{n}\right\}$ must contain a constant subsequence equal to $x$ everywhere, or it has a subsequence satisfying the hypotheses of Lemma 4.5. In the case of a constant subsequence, condition (b) translates to $\sigma_{n}=\sigma$ for all sufficiently large $n$ and this certainly implies convergence in $\operatorname{Stab}\left(X_{\beta}\right)^{\wedge}$. In the second case, we can appeal to Lemma 4.5. which allows us to combine Lemma 4.4 with Example 4.3 to show that $\left(x_{n}, \sigma_{n}\right) \rightarrow(x, \sigma)$ in $\left(B \otimes \mathcal{K}\left(L^{2}(G)\right)\right)_{\text {fix }, S}^{\wedge} \cong \operatorname{Stab}\left(X_{\beta}\right)^{\wedge}$.

We need a few more preliminaries before starting the proof of Theorem 4.2

Lemma 4.6. Suppose that $B$ is the section algebra of a continuous field of $C^{*}$ algebras over $X$, that $K$ is a compact subgroup of a second countable locally compact group $G$ and that $\beta: K \rightarrow$ Aut $B$ is an action of $K$ on $B$. Then the algebras $A_{\text {fix }}^{K}:=\left\{b \in B \otimes \mathcal{K}\left(L^{2}(K)\right): b(x) \in\left(B(x) \otimes \mathcal{K}\left(L^{2}(K)\right)\right)^{K_{x}, \beta^{x} \otimes \operatorname{Ad} \rho_{K}}\right\}$ and $A_{\text {fix }}^{G}:=$ $\left\{b \in B \otimes \mathcal{K}\left(L^{2}(G)\right): b(x) \in\left(B(x) \otimes \mathcal{K}\left(L^{2}(G)\right)\right)^{K_{x}, \beta^{x} \otimes \operatorname{Ad} \rho_{G}}\right\}$ are stably isomorphic.

Proof. We will show that $A_{\text {fix }}^{G} \cong A_{\text {fix }}^{K} \otimes \mathcal{K}\left(L^{2}(G / K)\right)$ when $G / K$ is equipped with the unique $G$-invariant measure such that $\int_{G} f(s) d s=\int_{G / K} \int_{K} f(s k) d k d \dot{s}$. Since $G$ is second countable, there is a locally bounded Borel section for the quotient map $q: G \rightarrow G / K$. Then $c$ induces a Borel isomorphism of $G / K \times K$ onto $G$ given by $(s K, k) \mapsto c(s) k$. In turn, this induces an isomorphism $\Phi$ : $L^{2}(G) \rightarrow L^{2}(G / K) \otimes L^{2}(K)$ given on continuous functions with compact support by $\Phi(\xi)(s K, k)=\xi(c(g) k)$. It is clear that $\Phi$ transforms the restriction of the right-regular representation $\rho_{G}$ to $K$ to $1 \otimes \rho_{K}$. Thus we get an isomorphism of $B \otimes \mathcal{K}\left(L^{2}(G)\right)$ onto $B \otimes \mathcal{K}\left(L^{2}(K)\right) \otimes \mathcal{K}\left(L^{2}(G / K)\right)$ which transforms $\left.\beta \otimes \operatorname{Ad} \rho_{G}\right|_{K}$ with $\beta \otimes \operatorname{Ad} \rho_{K} \otimes$ id. From this it is clear that $A_{\text {fix }}^{G} \cong A_{\text {fix }}^{K} \otimes \mathcal{K}\left(L^{2}(G / K)\right)$.

Remark 4.7. The second countability assumption in Lemma 4.6 is not necessary one always has a locally bounded Baire section and we could proceed as in [30, §4.5] - but the extra generality is unnecessary here, so we omit the details. 
Lemma 4.8. Suppose that $[\omega] \in H^{2}(K, \mathbb{T})$ for some compact group $K$ and let $\sigma: K \rightarrow U\left(\mathcal{H}_{\sigma}\right)$ be an $\omega$-representation of $K$. Suppose that $L$ is a closed subgroup of $K$ and $\rho: L \rightarrow U\left(\mathcal{H}_{\rho}\right)$ is an $\left.\omega\right|_{L}$-representation of $L$ such that $\rho \leq\left.\sigma\right|_{L}$. For any $k \in K$, let $k \cdot \rho: k L k^{-1} \rightarrow U\left(\mathcal{H}_{\sigma}\right)$ be given by $k \cdot \rho\left(k l k^{-1}\right)=\rho(l)$ for all $l \in L$. Then $k \cdot \rho$ is a $\left.\omega\right|_{k L k^{-1}-r e p r e s e n t a t i o n}$ with $k \cdot \rho \leq\left.\sigma\right|_{k L k^{-1}}$.

Proof. The result follows by applying the inner automorphism $C_{k}: K \rightarrow K$ given by $C_{k}(l)=k l k^{-1}$ to $\sigma$ and observing that $k \cdot \sigma=\sigma \circ C_{k}$ is equivalent to $\sigma$.

An action of a group on a space $X$ is said to have locally finitely many orbit types if for each $x \in X$ there is an open $G$-invariant neighborhood $U_{x}$ of $x$ and a finite set of conjugacy classes of subgroups of $G$ such that each stabilizer for the action of $G$ on $U_{x}$ lies in one of these conjugacy classes. A highly nontrivial but classical result (see, for example, [1, Chap. VI and VII]) implies that differentiable actions of compact Lie groups on manifolds have locally finitely many orbit types.

Lemma 4.9. Let $K$ be a second countable compact group acting on a separable stable continuous-trace $C^{*}$-algebra $B$ with spectrum $X$. Suppose that $K$ fixes $x \in X$ and that the action has finitely many orbit types. Let $\sigma \in \widehat{K}_{\omega_{x}}=\widehat{K}_{x, \omega_{x}}$. Then $\left(x_{n}, \sigma_{n}\right) \rightarrow(x, \sigma)$ in $\operatorname{Stab}\left(X_{\beta}\right)^{\wedge}$ if and only if

(a) $x_{n} \rightarrow x$ and

(b) there is an $N \in \mathbb{N}$ such that $\sigma_{x_{n}} \leq\left.\sigma\right|_{K_{x_{n}}}$ for all $n \geq N$.

Proof. Suppose that $\left(x_{n}, \sigma_{n}\right) \rightarrow(x, \sigma)$. Then we certainly have $x_{n} \rightarrow x$. If condition (b) fails, we can pass to a subsequence such that $\sigma_{x_{n}} \not \leq\left.\sigma\right|_{K_{x_{n}}}$ for all $n$. Since there are only finitely many orbit types, we can take this subsequence so that all the stability groups are conjugate. Thus, we can also assume that there are $s_{n} \in K$ such that $K_{s_{n} \cdot x}$ is a constant subgroup $\tilde{L}$. Since $K$ is compact, we can assume that $s_{n} \rightarrow s \in K$. Let $h_{n}=s^{-1} s_{n}$ and let $L=s \tilde{L} s^{-1}$. Since the action of conjugation on $\operatorname{Stab}\left(X_{\beta}\right)^{\wedge}$ is continuous, we see that $\left(h_{n} \cdot x_{n}, h_{n} \cdot \sigma_{n}\right):=\left(y_{n}, \rho_{n}\right) \rightarrow(x, \sigma)$. Passing to another subsequence, we assume that either $y_{n}=x$ for all $n$ or that $y_{n} \neq y_{m} \neq x$ for all $n \neq m$. Just as in the proof of Theorem 4.1 we can appeal to Lemma 4.5 to conclude that we eventually have $\left[\omega_{y_{n}}\right]=\left[\left.\omega_{x}\right|_{L}\right]$ and $\rho_{n} \leq\left.\sigma\right|_{L}$. But then Lemma 4.8 gives a contradiction.

Conversely, if (a) and (b) hold, then we can use similar arguments as in Theorem 4.1 to reduce to the situation of Lemma 4.5 and complete the proof.

Lemma 4.10. Suppose that $\beta: G \rightarrow$ Aut $B$ is a dynamical system such that there is a $G$-equivariant map $\phi: \widehat{B} \rightarrow G / H$. (Recall that the G-action on $\widehat{B}$ is given by $s \cdot \pi=\pi \circ \beta_{s}^{-1}$.) Let $Z:=\phi^{-1}(\{e H\}) \subseteq \widehat{B}$. Then $\widehat{B}$ is G-homeomorphic to $G \times{ }_{H} Z:=H \backslash(G \times Z)$ via the map $\psi$ sending $[s, \pi]$ to $s \cdot \pi$. (The action of $H$ on $G \times Z$ is given by $h \cdot(s, \pi)=\left(s h^{-1}, h \cdot \pi\right)$.)

Proof. This follows easily from [3, 4] or [30, Proposition 3.53]. (See [7, Theorem 6.2 and Corollary 6.3] for more details.)

Lemma 4.11. Suppose that $K$ is a compact group acting on a topological space $Y$, and suppose that $K$ fixes $y \in Y$. Let $\left\{y_{i}\right\}$ be a net in $Y$ such that $K \cdot y_{i} \rightarrow K \cdot y$ in $K \backslash Y$. Then the net $\left\{y_{i}\right\}$ converges to $y$ in $Y$.

Proof. If the assertion is false, then after passing to a subnet and relabeling, we can assume that there is a neighborhood $U$ of $y$ such that $y_{i} \notin U$ for all $i$. But 
since the orbit map is open, we may as well assume that there are $k_{i} \in K$ such that $k_{i} \cdot y_{i} \rightarrow y$. Since $K$ is compact, we can even assume that $k_{i} \rightarrow k$. But then $y_{i}=k_{i}^{-1} k_{i} \cdot y_{i} \rightarrow k^{-1} \cdot y=y$. Hence $y_{i}$ is eventually in $U$, which is a contradiction.

Remark 4.12 (Palais's Slice Property). As in [8, Definition 1.7], we say that a group $G$ acting properly on a locally compact space $X$ satisfies property (SP) (for Palais's slice property) if $X$ is locally induced from stabilizers in that each point $x \in X$ has a neigborhood $U_{x}$ such that there is a closed $G_{x}$-invariant set $S_{x} \subseteq U_{x}$ such that $x \in S_{x}$ and such that the induced space $G \times_{G_{x}} S_{x}$ is $G$-homeomorphic to $U_{x}$ via the map $[s, y] \mapsto s \cdot y$. Note that Lemma 4.11 implies that finding $S_{x}$ is equivalent to finding a continuous $G$-map $\phi_{x}: U_{x} \rightarrow G / G_{x}$ with $S_{x}=\phi_{x}^{-1}\left(\left\{e G_{x}\right\}\right)$. (In the case $U_{x}=G \times_{G_{x}} S_{x}$, we can define such a map by sending $[s, y] \mapsto s G_{x}$.) We call $S_{x}$ a local slice at $x$.

Palais's Slice Theorem ([20, Theorem 2.3.3]) implies that every proper action of a Lie group on a locally compact space has property (SP). Moreover, if $G$ acts differentiably on a manifold $X$, then $S_{x}$ can be taken to be a submanifold of $U_{x}$ such that the action of $G_{x}$ on $S_{x}$ is differentiable. This follows from the construction of the slice in [20, $\S 2.2]$ (combine the first lemma of [20, $\$ 2.2]$ with [20, Proposition 2.1.7]). Hence the action of $G_{x}$ on $S_{x}$, and therefore the action of $G$ on $X$, has locally finitely many orbit types.

Proof of Theorem 4.2. As in the proof of Theorem 4.1 we can assume that $B$ is stable. We let $\left\{\left(x_{n}, \sigma_{n}\right)\right\}$ be a sequence in $\operatorname{Stab}\left(X_{\beta}\right)^{\wedge}$ and $(x, \sigma) \in \operatorname{Stab}\left(X_{\beta}\right)^{\wedge}$ such that $x_{n} \rightarrow x$. In view of Remark 4.12 above, we can also assume that $X \cong G \times{ }_{G_{x}} Y$ for a local slice $Y$ at $x$ and that the corresponding action of $G_{x}$ on $Y$ has finitely many orbit types. Let $\phi=\phi_{x}: X \rightarrow G / G_{x}$ be the corresponding $G$-equivariant map. Then we get a $G$-equivariant map $\psi: \operatorname{Stab}\left(X_{\beta}\right)^{\wedge} \rightarrow G / G_{x}$ by $\psi(z, \rho)=$ $\phi(z)$. Let $Z:=\psi^{-1}\left(\left\{e G_{x}\right\}\right)$. Since $\operatorname{Stab}\left(X_{\beta}\right)^{\wedge}$ is $G$-equivariantly isomorphic to $\left(B \otimes \mathcal{K}\left(L^{2}(G)\right)\right)_{\text {fix }}^{\wedge}$, we obtain a $G$-homeomorphism

$$
\Phi: G \times_{G_{x}} Z \rightarrow \operatorname{Stab}\left(X_{\beta}\right)^{\wedge}
$$

given by $\Phi([s,(y, \rho)])=(s \cdot y, s \cdot \rho)$. Choose $s_{n} \in G$ and $\left(y_{n}, \rho_{n}\right) \in Z$ such that $\left(s_{n}^{-1} \cdot y_{n}, s_{n}^{-1} \cdot \rho\right)=\left(x_{n}, \sigma_{n}\right)$ for all $n$. Since $Y$ is a slice at $x$, the map $G_{x} \cdot y \mapsto G \cdot y$ is a homeomorphism of $G_{x} \backslash Y$ onto $G \backslash X$. Since $G \cdot x_{n} \rightarrow G \cdot x$ and $G \cdot y_{n}=G \cdot x_{n}$, we must have $G_{x} \cdot y_{n} \rightarrow G_{x} \cdot x$. Thus $y_{n} \rightarrow x$ by Lemma 4.11.

Assume now that $\left(x_{n}, \sigma_{n}\right) \rightarrow(x, \sigma)$ in $\operatorname{Stab}\left(X_{\beta}\right)^{\wedge}$. Then we certainly have $x_{n} \rightarrow x$, so we can assume the setup in the previous paragraph. Then we claim that $\left(y_{n}, \rho_{n}\right) \rightarrow(x, \sigma)$ in $\operatorname{Stab}\left(X_{\beta}\right)^{\wedge}$. Replacing $\left\{\left(y_{n}, \rho_{n}\right)\right\}$ by a subsequence, it suffices to see that a subsequence converges to $(x, \sigma)$. Since $y_{n} \rightarrow x$ and $x_{n}=s_{n}^{-1} \cdot y_{n} \rightarrow x$, the properness of the action allows us to pass to a subsequence and relabel and assume that $s_{n} \rightarrow s \in G_{x}$. Then it follows from the continuity of the $G$-action on $\operatorname{Stab}\left(X_{\beta}\right)^{\wedge}$ that $\left(y_{n}, \rho_{n}\right)=s_{n}^{-1} \cdot\left(x_{n}, \sigma_{n}\right) \rightarrow s^{-1} \cdot(x, \sigma)=(x, \sigma)$. This proves the claim.

We have $Z=\left(\left(B \otimes \mathcal{K}\left(L^{2}(G)\right)\right)_{\text {fix }, Y}\right)^{\wedge}$. On the other hand, $G_{x}$ acts on $\left.B\right|_{Y}$ and Lemma 4.6 implies that the corresponding space $\operatorname{Stab}\left(Y_{\beta}\right)^{\wedge} \cong\left(\left(B \otimes \mathcal{K}\left(L^{2}\left(G_{x}\right)\right)\right)_{\text {fix }}\right)^{\wedge}$ is homeomorphic to $Z$. Thus it follows from Lemma 4.9 that for large $n$ we have $\left[\omega_{s_{n} \cdot x_{n}}\right]=\left[\omega_{y_{n}}\right]=\left[\left.\omega_{x}\right|_{G_{x}}\right]=\left[\left.\omega_{x}\right|_{G_{s_{n} \cdot x_{n}}}\right]$ and $s_{n} \cdot \sigma_{n}=\rho_{n} \leq\left.\sigma\right|_{G_{s_{n} \cdot x_{n}}}$. Thus condition (b) holds and we've established the forward implication of the theorem. 
The converse follows by applying Lemma 4.4 and Lemma 4.5 as in the proof of Theorem 4.1 to the subsequence $\left\{\left(s_{l} \cdot y_{l}, s_{l} \cdot \rho_{l}\right)\right\}$. Since $G_{s_{l} \cdot y_{l}}=L \subseteq G_{x}$ and $s_{l} \cdot \rho_{l} \leq$ $\left.\sigma\right|_{L}$, it follows from those lemmas that $\left(s_{l} \cdot y_{l}, s_{l} \cdot \rho_{l}\right) \rightarrow(x, \sigma)$. Thus condition (b) tells us that every subsequence of $\left\{\left(x_{n}, \sigma_{n}\right)\right\}$ has a subsequence converging to $(x, \sigma)$. This implies $\left(x_{n}, \sigma_{n}\right) \rightarrow(x, \sigma)$ and completes the proof.

\section{Applichtion to group extensions}

In this section we want to study of the unitary dual $\widehat{G}$ of a locally compact group $G$ which fits into a short exact sequence

$$
1 \longrightarrow N \stackrel{\iota}{\longrightarrow} G \stackrel{q}{\longrightarrow} K \longrightarrow 1
$$

of locally compact groups in which $N$ is abelian and $K$ is compact. Following Green ([12] but see [6, Chapter 1] for a survey) we may write the $\mathrm{C}^{*}$-group algebra $C^{*}(G)$ as a twisted crossed product $C^{*}(N) \rtimes_{\alpha, \tau}(G, N) \cong C_{0}(\widehat{N}) \rtimes_{\widehat{\alpha}, \widehat{\tau}}(G, N)$ in which the action $\alpha$ is given by the conjugation action on the dense subalgebra $C_{c}(N) \subseteq C^{*}(N)$ and the twist $\tau: N \rightarrow U M\left(C^{*}(N)\right)$ is given by the canonical inclusion map. Then $(\widehat{\alpha}, \widehat{\tau})$ is the twisted action on $C_{0}(\widehat{N})$ corresponding to $(\alpha, \tau)$ via the Fourier isomorphism $C^{*}(N) \cong C_{0}(\widehat{N})$. Using a version of the Packer-Raeburn stabilization trick (e.g., see [6, Chapter 2]) we see that the twisted system is Morita equivalent to an action $\beta$ of the compact group $K=G / N$ on $B:=C_{0}(\widehat{N}, \mathcal{K})$ which covers the conjugation action of $K$ on $\widehat{N}$. Thus we are precisely in the situation of our general results.

Note that Morita equivalence of twisted actions induces a Morita equivalence of the twisted crossed products, hence a homeomorphism between the dual spaces of these crossed products. Moreover, it has been worked out in [6. Chapter 2] that passing to Morita equivalent twisted actions is compatible with the MackeyRieffel-Green machine of inducing representations from the stabilizers including the computation of the Mackey obstructions (see [6, Proposition 2.1.4 and 2.1.5]). Thus we see that $\widehat{G}$ is homeomorphic to $G \backslash \operatorname{Stab}\left(\widehat{N}_{\beta}\right)^{\wedge}$ with $\operatorname{Stab}\left(\widehat{N}_{\beta}\right)^{\wedge}$ topologized as in Definition 3.4

In order to get a description of the space $\operatorname{Stab}\left(\widehat{N}_{\beta}\right)^{\wedge}$ we need to compute the Mackey-obstructions of the twisted system $\left(C_{0}(\widehat{N}), G, N, \widehat{\alpha}, \widehat{\tau}\right)$. Let $c: K \rightarrow G$ be a fixed choice of a Borel section for the quotient map $q: G \rightarrow K$ such that $c(e N)=e$, where $e$ denotes the unit of $G$. Then, for each $\chi \in \widehat{N}$ we get a Borel extension $\tilde{\chi}: G \rightarrow \mathbb{T}$ of the character $\chi$ by putting

$$
\tilde{\chi}(s):=\chi\left(c(q(s))^{-1} s\right) .
$$

If we restrict this map to the stabilizer

$$
G_{\chi}=\left\{s \in G: \chi\left(s n s^{-1}\right)=\chi(n) \text { for all } n \in N\right\}
$$

of the character $\chi$ in $G$, then we can check that $\left(\epsilon_{\chi}, \tilde{\chi}\right)$, where $\epsilon_{\chi}: C_{0}(\widehat{N}) \rightarrow \mathbb{C}$ denotes evaluation at $\chi$, is an $\omega_{\chi}$-covariant representation of the twisted system $\left(C_{0}(\widehat{N}), G_{\chi}, N, \widehat{\alpha}, \widehat{\tau}\right)$, with multiplier $\omega_{\chi}$ given by

$$
\omega_{\chi}(s, t)=\tilde{\chi}(s) \tilde{\chi}(t) \tilde{\chi}(s t)^{-1} .
$$


We then compute

$$
\begin{aligned}
\omega_{\chi}(s, t) & =\chi\left(c(q(s))^{-1} s\right) \chi\left(c(q(t))^{-1} t\right) \chi\left(t^{-1} s^{-1} c(q(s t))\right) \\
& =\chi\left(c(q(s))^{-1} s\right) \chi\left(c(q(t))^{-1} s^{-1} c(q(s t))\right)
\end{aligned}
$$

which, since $\chi$ is invariant under conjugation with elements in $G_{\chi}$, is

$$
\begin{aligned}
& =\chi\left(c(q(s))^{-1} s\right) \chi\left(s^{-1} c(q(s t)) c(q(t))^{-1}\right) \\
& =\chi\left(c(q(s))^{-1} c(q(s t)) c(q(t))^{-1}\right) .
\end{aligned}
$$

Thus we see that $\omega_{\chi}$ factors through a cocycle on the stabilizer $K_{\chi}:=G_{\chi} / N$ of $\chi$ in $K$ and we obtain

$$
\operatorname{Stab}\left(\widehat{N}_{\beta}\right)^{\wedge}=\left\{(\chi, \sigma): \chi \in \widehat{N} \text { and } \sigma \in \widehat{K}_{\chi,\left[\omega_{\chi}\right]}\right\}
$$

with $\omega_{\chi}$ as in the above computations. Now if $K$ is a compact Lie group, we may apply Theorem 4.2 (or Theorem 4.1 if $K$ is finite) to obtain a description of the topology on $\operatorname{Stab}\left(\widehat{N}_{\beta}\right)^{\wedge}$ in terms of convergent sequences.

Example 5.1 (The group $G=\mathbf{p} 4 \mathbf{g}$ ). We want to illustrate the above procedure in the particular example of the crystallographic group $G=\mathbf{p} \mathbf{4} \mathbf{g}$, which is the subgroup of the full motion group $\mathbb{R}^{2} \rtimes \mathrm{O}(2)$ generated by $\left\{(n, E): n \in \mathbb{Z}^{2}\right\}$ together with the elements

$$
\left\{(0, R),\left(0, R^{2}\right),\left(0, R^{3}\right),(v, S),(v, S R),\left(v, S R^{2}\right),\left(v, S R^{3}\right)\right\},
$$

where $R=\left(\begin{array}{cc}0 & -1 \\ 1 & 0\end{array}\right), S=\left(\begin{array}{cc}1 & 0 \\ 0 & -1\end{array}\right)$ and $v=\left(\begin{array}{c}1 / 2 \\ 1 / 2\end{array}\right) \in \mathbb{R}^{2}$. Then $G$ fits into an extension

$$
0 \longrightarrow \mathbb{Z}^{2} \longrightarrow G \longrightarrow D_{4} \longrightarrow 0
$$

where $D_{4}=\left\{E, R, R^{2}, R^{3}, S, S R, S R^{2}, S R^{3}\right\}$ is the dihedral group. The quotient map is given by projection on the second factor and we have an obvious section $c: D_{4} \rightarrow G$ given by $c(X)=(0, X)$ if $X \in\langle R\rangle$ and $c(X)=(v, X)$ if $X \in S\langle R\rangle$. The conjugation action of $D_{4}=G / \mathbb{Z}^{2}$ on $\mathbb{T}^{2}=\widehat{\mathbb{Z}^{2}}$ is given by matrix multiplication; that is, if we write

then we have

$$
\exp _{2}: \mathbb{R}^{2} \rightarrow \mathbb{T}^{2} ; \exp _{2}\left(\begin{array}{c}
s \\
t
\end{array}\right)=\left(\begin{array}{c}
e^{2 \pi i s} \\
e^{2 \pi i t}
\end{array}\right)
$$

$$
X \cdot \exp _{2}\left(\begin{array}{l}
s \\
t
\end{array}\right)=\exp _{2}\left(X\left(\begin{array}{l}
s \\
t
\end{array}\right)\right)
$$

for $X \in D_{4}, s, t \in \mathbb{R}$. This action has been studied in [8] (see Examples 2.6, 3.5 and 4.10 of that paper). In particular, it is shown in [8] that the image under the exponential map of the triangle

$$
Z:=\left\{\left(\begin{array}{l}
s \\
t
\end{array}\right) \in \mathbb{R}^{2}: 0 \leq t \leq \frac{1}{2} \text { and } 0 \leq s \leq t\right\}
$$

is a topological fundamental domain for the action of $D_{4}$ on $\mathbb{T}^{2}$ in the sense that the mapping $Z \rightarrow D_{4} \backslash \mathbb{T}^{2} ; z \mapsto D_{4} z$ is a homeomorphism. It follows from this that

$$
\operatorname{Stab}(Z)^{\wedge}=\left\{\left(\chi_{z}, \sigma\right): z \in Z, \sigma \in \widehat{D}_{z,\left[\omega_{z}\right]}\right\}
$$

is a topological fundamental domain for $\operatorname{Stab}\left(\mathbb{T}_{\beta}^{2}\right)^{\wedge}$ for the action $\beta$ of $D_{4}$ on $C\left(\mathbb{T}^{2}, \mathcal{K}\right)$ as described in the discussion above, where we write $\chi_{z}: \mathbb{Z}^{2} \rightarrow \mathbb{T}$ for the character $\chi_{z}(n)=e^{2 \pi i\langle z, n\rangle}$ for $z \in \mathbb{R}^{2}, D_{z}$ for the stabilizer of $\chi_{z}$ in $D_{4}$ and $\omega_{z}$ for the Mackey-obstruction at $\chi_{z}$ as in (5.1). It therefore follows from Theorem 3.5 and the above considerations that $\widehat{G}$ is homeomorphic to $\operatorname{Stab}(Z)^{\wedge}$ (which we regard as a closed subset of $\left.\operatorname{Stab}\left(\mathbb{T}_{\beta}^{2}\right)^{\wedge}\right)$. 
We want to study the $\operatorname{space} \operatorname{Stab}(Z)^{\wedge}$ more closely. For this we first note that the action of $D_{4}$ on $Z$ (or rather its image in $\mathbb{T}^{2}$ ) has the following stabilizers:

- $D_{\left(\begin{array}{l}s \\ t\end{array}\right)}=\{E\}$ if $0<s<t<\frac{1}{2}$,

- $D_{\left(\begin{array}{l}s \\ s\end{array}\right)}=\left\langle S R^{3}\right\rangle=: K_{1}$ if $0<s<\frac{1}{2}$,

- $D_{\left(\begin{array}{l}0 \\ t\end{array}\right)}=\left\langle S R^{2}\right\rangle=: K_{2}$ if $0<t<\frac{1}{2}$,

- $D_{\left(\begin{array}{c}s \\ 1 / 2\end{array}\right)}=\langle S\rangle=: K_{3}$ if $0<s<\frac{1}{2}$,

- $D_{\left(\begin{array}{c}0 \\ 1 / 2\end{array}\right)}=\left\langle S, R^{2}\right\rangle=: H$, and

- $D_{\left(\begin{array}{l}0 \\ 0\end{array}\right)}=D_{\left(\begin{array}{l}1 / 2 \\ 1 / 2\end{array}\right)}=D_{4}$.

We need to compute the cocycles $\omega_{z}:=\omega_{\chi z}$ on the stabilizers $D_{z}$ and the $\omega_{z^{-}}$ representations of $D_{z}$ for all $z \in Z$. Clearly, for $z$ in the interior $Z^{\circ}$ of $Z$ we have trivial stabilizers and the trivial representations on these stabilizers. As a consequence, the portion of $\operatorname{Stab}(Z)^{\wedge}$ corresponding to $Z^{\circ}$ is homeomorphic to $Z^{\circ}$.

To study the boundary points, we first observe that the cocycle $\partial c \in Z^{2}\left(D_{4}, \mathbb{Z}^{2}\right)$ for the cross-section $c: D_{4} \rightarrow G$ can be computed as

$$
\partial c(X, Y)=c(X)^{-1} c(X Y) c(Y)^{-1}= \begin{cases}0 & \text { if } Y \in\langle R\rangle, \\ X^{-1} v-v & \text { if } Y \in S\langle R\rangle, X \in\langle R\rangle, \\ -\left(X^{-1} v+v\right) & \text { if } Y, X \in S\langle R\rangle .\end{cases}
$$

If we plug this into the characters $\chi_{z}$ we get the following cocycles on the stabilizers:

Case 1 . On the interiors of the edges of $Z$ with stabilizers $K_{1}, K_{2}, K_{3}$ we get the values

- $\omega_{\left(\begin{array}{c}s \\ s\end{array}\right)}\left(S R^{3}, S R^{3}\right)=e^{-4 \pi i s}$ on $K_{1}=\left\langle S R^{3}\right\rangle, 0<s<\frac{1}{2}$;

- $\omega_{\left(\begin{array}{l}0 \\ t\end{array}\right)}\left(S R^{2}, S R^{2}\right)=e^{-2 \pi i t}$ on $K_{2}=\left\langle S R^{2}\right\rangle, 0<t<\frac{1}{2}$;

- $\omega_{\left(\begin{array}{c}s \\ \frac{1}{2}\end{array}\right)}(S, S)=e^{2 \pi i s}$ on $K_{3}=\langle S\rangle, 0<s<\frac{1}{2}$

(and all other values 1 ). Note that these cocycles, regarded as $\mathbb{T}$-valued cocycles, are cohomologous to the trivial one. In fact, if $u$ is any element of $\mathbb{T}$, if $\epsilon$ is the generator of $\mathbb{Z} / 2 \mathbb{Z}$, and if $\omega^{u}$ is the the $\mathbb{T}$-valued cocycle given by $\omega^{u}(\epsilon, \epsilon)=u$ (and all other values 1 ), then $\omega^{u}=\partial \mu_{w}$ if $w \in \mathbb{T}$ is a square root of $u$ and $\mu_{w}: \mathbb{Z} / 2 \mathbb{Z} \rightarrow \mathbb{T}$ is given by $\mu_{w}(1)=1$ and $\mu_{w}(\epsilon)=w$. It follows in particular that if $w_{1}, w_{2}$ are the two roots of $u$, then $\mu_{w_{1}}, \mu_{w_{2}}$ are the two irreducible $\omega^{u}$-representations of $\mathbb{Z} / 2 \mathbb{Z}$. This general description can be applied to the cocycles on the groups $K_{1}, K_{2}, K_{3}$ considered above.

It then follows from Theorem 4.1 that on each open line segment of $\partial Z$ the corresponding portion of $\operatorname{Stab}(Z)^{\wedge}$ is homeomorphic to $(0,1) \times\{1,-1\}$. For example, on the segment $S_{1}:=\left\{\left(\begin{array}{l}s \\ s\end{array}\right): 0<s<\frac{1}{2}\right\}$ the homeomorphism $(0,1) \times\{1,-1\} \rightarrow$ $\operatorname{Stab}\left(S_{1}\right)^{\wedge}$ is given by $(t, \pm 1) \mapsto\left(\left(\frac{t}{2}, \frac{t}{2}\right), \mu_{t, \pm 1}\right)$ with $\mu_{t, \pm 1}\left(S R^{3}\right)= \pm e^{\pi i t}$ and similarly on $S_{2}:=\left\{\left(\begin{array}{l}0 \\ t\end{array}\right): 0<t<\frac{1}{2}\right\}$ and $S_{3}:=\left\{\left(\begin{array}{c}s \\ \frac{1}{2}\end{array}\right): 0<s<\frac{1}{2}\right\}$.

Case 2. For $z_{0}=\left(\begin{array}{l}0 \\ 0\end{array}\right)$ we get the trivial cocycle $\omega_{z_{0}} \equiv 1$ on $D_{4}$ and the ordinary unitary dual of $D_{4}$ which consists of the representations $\left\{\mu_{0}, \mu_{1}, \mu_{2}, \mu_{3}, \lambda\right\}$ in which $\left\{\mu_{0}, \mu_{1}, \mu_{2}, \mu_{3}\right\}$ denote the characters of the commutative quotient $D_{4} /\left\langle R^{2}\right\rangle \cong\langle\tilde{R}\rangle \times$ 
$\langle\tilde{S}\rangle$ (where $\tilde{R}$ and $\tilde{S}$ denote the images of $R$ and $S$ in $D_{4} /\left\langle R^{2}\right\rangle$ ) and $\lambda: D_{4} \rightarrow M_{2}(\mathbb{C})$ denotes the representation given by the inclusion $D_{4} \subseteq U(2)$.

Case 3. For $z_{1}:=\left(\begin{array}{c}0 \\ 1 / 2\end{array}\right)$ and $D_{z_{1}}=H=\left\langle R^{2}, S\right\rangle$ we get the values

$$
\omega_{z_{1}}\left(R^{2}, Y\right)=\omega_{z_{1}}\left(S R^{2}, Y\right)=-1
$$

for $Y \in\left\{S, S R^{2}\right\}$ and 1 for all other values. Since $\omega_{z_{1}}$ takes its values in $C_{2}:=$ $\{1,-1\}$, we may use the central extension $H \times{ }_{\omega_{z_{1}}} C_{2}$ which, as a set, is the direct product $H \times C_{2}$ with multiplication given by

$$
(X, u)(Y, v)=\left(X Y, \omega_{z_{1}}(X, Y) u v\right)
$$

for the study of the $\omega_{z_{1}}$-representations of $H$. In fact, there is a one-to-one correspondence between the irreducible $\omega_{z_{1}}$-representations $\sigma$ of $H$ and the irreducible unitary representations $\tilde{\sigma}$ of $H \times{ }_{\omega_{z_{1}}} C_{2}$ which satisfy $\tilde{\sigma}(E,-1)=-1$ given as follows: if $\sigma \in \widehat{H}_{\left[\omega_{z_{1}}\right]}$ is given, the corresponding representation $\tilde{\sigma}$ is given by $\tilde{\sigma}(X, u)=u \sigma(X)$, and if $\tilde{\sigma}$ is given, then $\sigma(X)=\tilde{\sigma}(X, 1)$ is the corresponding projective representation of $H$.

Now a short computation shows that $D_{4}$ is isomorphic to $H \times{ }_{\omega_{z_{1}}} C_{2}$ by sending $R$ to $\left(S R^{2}, 1\right)$ and $S$ to $\left(R^{2}, 1\right)$. If we then identify $\widehat{D}_{4}$ with $\left(H \times{ }_{\omega_{z_{1}}} C_{2}\right)^{\wedge}$ via this isomorphism, we see that only the two-dimensional representation $\lambda$ of $D_{4}$ corresponds to a representation of $H \times{ }_{\omega_{z_{1}}} C_{2}$ which takes value -1 on $(E,-1)$. So we only get one (class) $[\sigma]$ of irreducible $\omega_{z_{1}}$-representations of $H$ given by $\sigma: H \rightarrow U(2)$,

$$
\sigma(E)=1, \quad \sigma\left(R^{2}\right)=\left(\begin{array}{cc}
1 & 0 \\
0 & -1
\end{array}\right), \quad \sigma(S)=\left(\begin{array}{ll}
0 & 1 \\
1 & 0
\end{array}\right) \quad \text { and } \quad \sigma\left(S R^{2}\right)=\left(\begin{array}{cc}
0 & -1 \\
1 & 0
\end{array}\right) .
$$

Case 4. Finally, for $z_{2}=\left(\begin{array}{l}1 / 2 \\ 1 / 2\end{array}\right)$ we get the cocycle $\omega_{z_{2}}$ on $D_{4}$ with values

$$
\omega_{z_{2}}(R, Y)=\omega_{z_{2}}\left(R^{3}, Y\right)=\omega_{z_{2}}(S, Y)=\omega_{z_{2}}\left(S R^{2}, Y\right)=-1
$$

for all $Y \in S\langle R\rangle$ and 1 otherwise. To study the $\omega_{z_{2}}$-representations of $D_{4}$ we proceed as above by studying the central extension $L:=D_{4} \times{ }_{\omega_{z_{2}}} C_{2}$ and determine those representations $\tau \in \widehat{L}$ which satisfy $\tau(E,-1)=-1$. Let's denote this set by $\widehat{L}^{-}$. One checks that $L$ is generated by the elements $(R, 1)$ and $(S, 1)$, and with a little work we see that $\widehat{L}^{-}$contains

- four one-dimensional representations $\zeta_{0}, \ldots, \zeta_{3}$ given on these generators by

$$
\begin{aligned}
& \zeta_{0}(R, 1)=\zeta_{0}(S, 1)=i, \\
& \zeta_{1}(R, 1)=-\zeta_{1}(S, 1)=i, \\
& \zeta_{2}(R, 1)=-\zeta_{2}(S, 1)=-i, \\
& \zeta_{3}(R, 1)=\zeta_{3}(S, 1)=-i \text { and }
\end{aligned}
$$

- one two-dimensional representation $\tau: L \rightarrow U(2)$ given on the generators by

$$
\tau(R, 1)=S=\left(\begin{array}{rr}
1 & 0 \\
0 & -1
\end{array}\right) \quad \text { and } \quad \tau(S, 1)=R=\left(\begin{array}{rr}
0 & -1 \\
1 & 0
\end{array}\right)
$$

Restricting these representations to $D_{4} \times\{1\} \subseteq L$ gives the desired $\omega_{z_{2}}$-representations of $D_{4}$ (which we shall call by the same letters below). 
By the above computations together with Theorem 4.1 we get the following description of $\operatorname{Stab}(Z)^{\wedge} \cong \widehat{G}$ together with its topology: As a set we have the disjoint union

$$
\begin{aligned}
\operatorname{Stab}(Z)^{\wedge}= & Z^{\circ} \amalg\left(S_{1} \amalg S_{2} \amalg S_{3}\right) \times\{1,-1\} \\
& \amalg\left\{z_{0}\right\} \times\left\{\mu_{0}, \ldots, \mu_{4}, \lambda\right\} \\
& \amalg\left\{z_{1}\right\} \times\{\sigma\} \\
& \amalg\left\{z_{2}\right\} \times\left\{\zeta_{0}, \ldots, \zeta_{3}, \tau\right\}
\end{aligned}
$$

with topology restricted to the single components the usual one. Moreover, $Z^{\circ}$ is open in $\operatorname{Stab}(Z)^{\wedge}$, and if we approach any boundary point $\bar{z} \in \partial Z$ by a sequence $\left(z_{n}\right)_{n} \in Z^{\circ}$, then this sequence converges to every element in $\operatorname{Stab}(Z)^{\wedge}$ which corresponds to $\bar{z}$.

Moreover, $\left(S_{1} \amalg S_{2} \amalg S_{3}\right) \times\{1,-1\}$ is open in $\operatorname{Stab}(\partial Z)^{\wedge}$. Assume that $\left(z_{n}, u_{n}\right)_{n}$ is a sequence in this set such that $z_{n}$ converges to one of the vertices $z_{0}, z_{1}, z_{2}$. By passing to a subsequence, if necessary, we may assume that $u_{n}= \pm 1$ is constant. Using Theorem 4.1 it is then very easy to describe the limit points of this sequence in $\operatorname{Stab}(Z)^{\wedge}$.

We will work this out for the case where $z_{n}=\left(\begin{array}{c}s_{n} \\ \frac{1}{2}\end{array}\right) \in S_{3}$ and we leave the other cases to the reader. The identification $S_{3} \times\{1,-1\} \cong \operatorname{Stab}\left(S_{3}\right)^{\wedge}$ is given by the map $\left(\left(\begin{array}{c}s \\ \frac{1}{2}\end{array}\right), \pm 1\right) \mapsto\left(\left(\begin{array}{c}s \\ \frac{1}{2}\end{array}\right), \mu_{s, \pm 1}\right)$ with $\mu_{s, \pm 1}(S)= \pm e^{\pi i s}$. Since $\mu_{\frac{1}{2}, \pm 1}(S)=e^{\pi i \frac{1}{2}}= \pm i$ we easily see that $\mu_{\frac{1}{2}, 1}=\left.\zeta_{0}\right|_{K_{3}}=\left.\zeta_{2}\right|_{K_{3}}$ and $\mu_{\frac{1}{2},-1}=\left.\zeta_{1}\right|_{K_{3}}=\left.\zeta_{3}\right|_{K_{3}}$. Moreover, $\tau(S)=R$ has the eigenvalues \pm 1 , so that both, $\mu_{\frac{1}{2}, 1}$ and $\mu_{\frac{1}{2},-1}$ are subrepresentations of $\left.\tau\right|_{K_{3}}$. Thus, we see that the sequence $\left(\left(\begin{array}{c}s_{n} \\ \frac{1}{2}\end{array}\right), \mu_{s_{n}, 1}\right)_{n}$ converges to $\left(z_{2}, \zeta_{0}\right),\left(z_{2}, \zeta_{2}\right)$ and $\left(z_{2}, \tau\right)$, and $\left(\left(\begin{array}{c}s_{n} \\ \frac{1}{2}\end{array}\right), \mu_{s_{n}, 1}\right)_{n}$ converges to $\left(z_{2}, \zeta_{1}\right),\left(z_{2}, \zeta_{3}\right)$ and $\left(z_{2}, \tau\right)$.

Similarly, if $s_{n} \rightarrow 0$, then $\mu_{0, \pm 1}(S)= \pm 1$. If $\sigma$ is the unique irreducible $\omega_{z_{1}}$ representation, we have $\sigma(S)=\left(\begin{array}{ll}0 & 1 \\ 1 & 0\end{array}\right)$ which has eigenvalues \pm 1 . Thus we see that $\mu_{0,1}$ and $\mu_{0,-1}$ are both subrepresentations of $\left.\sigma\right|_{K_{2}}$, hence both sequences $\left(\left(\begin{array}{c}s_{n} \\ \frac{1}{2}\end{array}\right), \mu_{s_{n}, \pm 1}\right)_{n}$ converge to $\left(z_{1}, \sigma\right)$.

\section{REFERENCES}

[1] Armand Borel, Seminar on transformation groups, With contributions by G. Bredon, E. E. Floyd, D. Montgomery, R. Palais. Annals of Mathematics Studies, No. 46, Princeton University Press, Princeton, N.J., pp. vii+245, 1960.

[2] Anton Deitmar and Siegfried Echterhoff, Principles of harmonic analysis, Universitext, Springer, New York, pp. xvi+333, 2009.

[3] Siegfried Echterhoff, On induced covariant systems, Proc. Amer. Math. Soc. 108 (1990), 703-706, DOI 10.2307/2047790.

[4] Siegfried Echterhoff, Erratum to: "On induced covariant systems" [Proc. Amer. Math. Soc. 108 (1990), no. 3, 703-706; MR 90f:46105], Proc. Amer. Math. Soc. 116 (1992), 581.

[5] Siegfried Echterhoff, The primitive ideal space of twisted covariant systems with continuously varying stabilizers, Math. Ann. 292 (1992), 59-84, DOI 10.1007/BF01444609.

[6] Siegfried Echterhoff, Crossed products with continuous trace, Mem. Amer. Math. Soc. 123 (1996), viii+134, DOI 10.1090/memo/0586.

[7] Siegfried Echterhoff, Crossed products, the Mackey-Rieffel-Green machine and applications, preprint, 2011. (arXiv:math.OA.1006.4975v2).

[8] Siegfried Echterhoff and Heath Emerson, Structure and K-theory of crossed products by proper actions, Expo. Math. 29 (2011), 300-344, DOI 10.1016/j.exmath.2011.05.001. 
[9] Siegfried Echterhoff and Jonathan Rosenberg, Fine structure of the Mackey machine for actions of abelian groups with constant Mackey obstuction, Pacific J. Math. 170 (1995), 1752.

[10] Siegfried Echterhoff and Dana P. Williams, Crossed products whose primitive ideal spaces are generalized trivial $\hat{G}$-bundles, Math. Ann. 302 (1995), 269-294, DOI 10.1007/BF01444496.

[11] Siegfried Echterhoff and Dana P. Williams, Locally inner actions on $C_{0}(X)$-algebras, J. Operator Theory 45 (2001), 131-160.

[12] Philip Green, The local structure of twisted covariance algebras, Acta Math. 140 (1978), 191-250.

[13] Astrid an Huef, Iain Raeburn, and Dana P. Williams, An equivariant Brauer semigroup and the symmetric imprimitivity theorem, Trans. Amer. Math. Soc. 352 (2000), 4759-4787.

[14] Steven Hurder, Dorte Olesen, Iain Raeburn, and Jonathan Rosenberg, The Connes spectrum for actions of abelian groups on continuous-trace algebras, Ergodic Theory Dynam. Systems 6 (1986), 541-560, DOI 10.1017/S0143385700003680.

[15] Eberhard Kirchberg and Simon Wassermann, Operations on continuous bundles of $C^{*}$ algebras, Math. Ann. 303 (1995), 677-697.

[16] Calvin C. Moore, Extensions and low dimensional cohomology theory of locally compact groups. I, Trans. Amer. Math. Soc. 113 (1964), 40-63.

[17] Calvin C. Moore, Extensions and low dimensional cohomology theory of locally compact groups. II, Trans. Amer. Math. Soc. 113 (1964), 64-86.

[18] Katharina Neumann, A description of the Jacobson topology on the spectrum of a transformation group $C^{*}$-algebras by proper actions, Ph.D. Thesis, Westfälische Wilhelms-Universität Münster, 2011.

[19] Dorte Olesen and Iain Raeburn, Pointwise unitary automorphism groups, J. Funct. Anal. 93 (1990), 278-309, DOI 10.1016/0022-1236(90)90130-D.

[20] Richard S. Palais, On the existence of slices for actions of non-compact Lie groups, Ann. of Math. 73 (1961), 295-323.

[21] John Phillips and Iain Raeburn, Crossed products by locally unitary automorphism groups and principal bundles, J. Operator Theory 11 (1984), 215-241.

[22] Iain Raeburn, Induced $C^{*}$-algebras and a symmetric imprimitivity theorem, Math. Ann. 280 (1988), 369-387, DOI 10.1007/BF01456331.

[23] Iain Raeburn and Jonathan Rosenberg, Crossed products of continuous-trace $C^{*}$-algebras by smooth actions, Trans. Amer. Math. Soc. 305 (1988), 1-45, DOI 10.2307/2001039.

[24] Iain Raeburn and Dana P. Williams, Pull-backs of $C^{*}$-algebras and crossed products by certain diagonal actions, Trans. Amer. Math. Soc. 287 (1985), 755-777, DOI 10.2307/1999675.

[25] Iain Raeburn and Dana P. Williams, Crossed products by actions which are locally unitary on the stabilisers, J. Funct. Anal. 81 (1988), 385-431, DOI 10.1016/0022-1236(88)90106-1.

[26] Iain Raeburn and Dana P. Williams, Moore cohomology, principal bundles, and actions of groups on $C^{*}$-algebras, Indiana Univ. Math. J. 40 (1991), 707-740, DOI 10.1512/iumj.1991.40.40032.

[27] Marc A. Rieffel, Proper actions of groups on $C^{*}$-algebras, Mappings of operator algebras (Philadelphia, PA, 1988), Progr. Math., vol. 84, Birkhäuser Boston, Boston, MA, 1990, pp. 141-182.

[28] Marc A. Rieffel, Integrable and proper actions on $C^{*}$-algebras, and square-integrable representations of groups, Expo. Math. 22 (2004), 1-53, DOI 10.1016/S0723-0869(04)80002-1.

[29] I. Schochetman, The dual topology of certain group extensions, Adv. in Math. 35 (1980), 113-128, DOI 10.1016/0001-8708(80)90044-4.

[30] Dana P. Williams, Crossed products of $C^{*}$-algebras, Mathematical Surveys and Monographs, vol. 134, American Mathematical Society, Providence, RI, 2007.

Mathematisches Institut, Westfälische Wilhelms-Universität Münster, Einsteinstr. 62 D-48149 Münster, Germany

E-mail address: echters@uni-muenster.de

Department of Mathematics, Dartmouth College, Hanover, New Hampshire 037553551

E-mail address: dana.williams@Dartmouth.edu 